\title{
A New Spherical Harmonics Scheme for Multi-Dimensional Radiation Transport I: Static Matter Configurations.
}

\author{
David Radice $^{\mathrm{a}}$, Ernazar Abdikamalov ${ }^{\mathrm{b}}$, Luciano Rezzolla ${ }^{\mathrm{a}, \mathrm{c}}$, Christian D. Ott ${ }^{\mathrm{b}}$ \\ ${ }^{a}$ Max Planck Institute für Gravitationsphysik, Albert Einstein Institute, Potsdam, Germany \\ ${ }^{b}$ TAPIR, California Institute of Technology, Pasadena, CA \\ ${ }^{c}$ Department of Physics and Astronomy, Louisiana State University, Baton Rouge, Louisiana, USA
}

\begin{abstract}
Recent work by McClarren \& Hauck 31 suggests that the filtered spherical harmonics method represents an efficient, robust, and accurate method for radiation transport, at least in the two-dimensional (2D) case. We extend their work to the three-dimensional (3D) case and find that all of the advantages of the filtering approach identified in 2D are present also in the 3D case. We reformulate the filter operation in a way that is independent of the timestep and of the spatial discretization. We also explore different second- and fourth-order filters and find that the second-order ones yield significantly better results. Overall, our findings suggest that the filtered spherical harmonics approach represents a very promising method for 3D radiation transport calculations.
\end{abstract}

Keywords: Radiation transport, $P_{N}-$ method, spherical harmonics, asymptotic diffusion limit, discontinuous Galerkin

\section{Introduction}

Many phenomena in the universe involve the transport of radiation and need to be modeled with radiation-transport techniques that are as accurate as possible to maximize the match with observations. Examples are nova and supernova explosions, gamma-ray bursts, star or planet formation, luminous blue variable outbursts, stellar winds, etc. In these examples, radiation plays a major role in exchanging energy and/or momentum between different parts of the system. In most of these cases, the radiation is composed of photons, but the radiation can also be composed of neutrinos in studies of core-collapse supernova explosion mechanisms (see, e.g., 6, 27) or in modeling the torus orbiting the black hole produced in the merger of neutron stars (see, e.g., 44]).

One of the main difficulties in solving the radiation-transport equation arises from the multidimensionality of the problem. Radiation is described not only by the position of the radiation carriers, but also by their direction of propagation and energy (or, equivalently, by their momentum), making the problem $(6+1)$ dimensional in the most general case ( 3 dimensions for the spatial coordinates, 2 for the angular direction, 1 for the energy, and 1 for the time). Another source of complication stems from the fact that many problems consist of regions of strongly-varying optical depth. For example, many astrophysical systems contain a central source of radiation, where the optical depth is high, surrounded by more transparent outer regions. Due to high opacity near the center, radiation migrates out of that region mostly via diffusion, while in the outer parts, it streams to infinity more freely without much interaction with matter. Correspondingly, in the limit of infinite optical depth, the transport equation acquires a parabolic character, while it maintains a hyperbolic one in all other regimes (see, e.g., [35]). Radiation-transport approaches must therefore handle accurately not only these two distinct regimes, but also the transition between the two, which is generally the most difficult to treat. These features make the solution of the transport equation both complex and computationally expensive.

There are two commonly used ways of simplifying the solution of the transport equation. One approach consists in reducing the number of degrees of freedom by assuming spherical or axial symmetry. While this is 
a good simplification for some problems, there are many other situations in which the system does not posses any spatial symmetries and hence the transport equations need to be solved in three spatial dimensions. Another way of simplifying the problem is via approximating the form of the transport equation (this is equivalent to reducing dimensionality in the momentum space). One of the simplest examples is the diffusion approximation, where one approximates the transport equation with the diffusion equation (e.g., [41]). This makes the equation far simpler and computationally less expensive to solve (e.g., [35]). However, there is a price to pay for this: Although the diffusion approximation is accurate at high optical depth, it leads to incorrect results in low optical depth, i.e. "transparent", regions. This can be improved by using flux limiters (e.g., [16]), but the diffusion approximation still cannot correctly capture the anisotropy of radiation commonly encountered in those regions (see, e.g., [40]). Moreover, the treatment of semitransparent regimes is somewhat artificial because the radiation fluxes in those regions are usually calculated via interpolation between the two fluxes calculated assuming free-streaming and diffusive transport. There are several more accurate ways of approximating the transport (e.g., two-moment schemes with analytic closures, etc.; e.g., 9]), but the solution of the full $(6+1)$-dimensional transport equation is often not only desirable, but actually necessary for an accurate modeling.

One of the most commonly used methods for solving the transport equation in the multidimensional (both in space and momentum) case is the discrete-ordinate $\left(S_{N}\right)$ method, which solves the transport equation along several directions in each spatial zone [16, 40, 46, 22. Unfortunately, this method has several drawbacks. Most importantly, it suffers from "ray effects" in multidimensional calculations [36]. Due to the discrete nature of the angular representation, in fact, radiation cannot reach regions between these discrete directions, leading to large spatial oscillations in the transport variables. In addition, time-implicit $S_{N}$ methods require very complex solutions and parallelization procedures 5$]$.

Monte-Carlo methods (e.g., 20, 21, 3]) are often regarded as the most accurate method for radiation transport, but they are also not without drawbacks: Monte-Carlo solutions exhibit statistical noise due to the finite sampling of the phase space. Since this noise decreases only as $N^{-1 / 2}$, where $N$ is the number of Monte Carlo particles, it can take many particles to produce a sufficiently smooth solution, making large simulations computationally very expensive.

Another approach to radiation transport is the spherical harmonics, or $P_{N}$, method. This method is based on an expansion of the radiation intensity (or distribution function) in angles using spherical harmonics basis functions. This results in a hyperbolic system of partial differential equations for the expansion coefficients, which represent angular moments in this basis. The spherical harmonics method has several interesting characteristics. Due to hyperbolicity, the $P_{N}$ equations approximate radiation as a series of waves with velocity bounded by the speed of light 32 . This restriction is consistent with the transport equation, in contrast to diffusion methods where radiation propagates at infinite velocity. Moreover, the spherical harmonics expansion exhibits formal spectral convergence to the true solution. Such an expansion also preserves the rotational invariance of the solution, unlike $S_{N}$ methods, where the absence of such invariance leads to the ray effects mentioned above ${ }^{1}$ Another advantage of the $P_{N}$ method is that it generally uses less memory to model a given angular distribution at a given accuracy compared to, e.g., the $S_{N}$ method. A $P_{N}$ truncation is roughly equivalent to a $S_{N+1}$ solution, but, while the former has $(N+1)^{2}$ degrees of freedom, the latter has $2(N+1)^{2}$, thus it requires roughly twice as much memory. Given that memory requirements represent one of the main difficulties in $3 \mathrm{D}$ radiation-transport calculations, a factor two in memory saving represents a significant advantage.

However, spherical harmonics methods also have some negative aspects. Most importantly, in transparent regions, the solution to the $P_{N}$ hyperbolic system exhibits non-physical oscillations. These oscillations are related to the so-called Gibbs phenomenon that occurs when non-smooth functions are approximated with smooth basis functions [8]. The worst consequence of such oscillations is that they can cause the radiation intensity to become negative, which may lead to negative matter temperatures when the radiation

\footnotetext{
${ }^{1}$ Here, rotational invariance means that the operators of angular discretization and of rotation in space are commutative. In other words, the result of any rotation and then of a spherical harmonics angular discretization is the same as that of an angular discretization and then of a rotation [8]. In the $S_{N}$ method, this is true only for those rotations which map the angular grid onto itself.
} 
transport is coupled to the matter energy equation 32, 37. There have been several attempts to address this problem [39, 37, 10, 31, 38. One of the most efficient, robust, and accurate approaches is the one by McClarren \& Hauck [31, in which filters are proposed to remove oscillations of the radiation intensity. By filtering out the oscillations, McClarren \& Hauck 31] were able to avoid negative solutions while maintaining high angular accuracy. Their filtering approach also has the advantage of being easily extendable to highorder $P_{N}$ expansions, producing formal convergence to the transport solution and preserving the equilibrium diffusion limit.

Although the results of [31] strongly suggest the idea of applying a filter to spherical harmonics expansions is an efficient, robust, and accurate way of doing $P_{N}$ radiation transport, there remain several open questions. For example, 31] considered only one type of filter, the so-called spherical-spline expansion [8]. There are other types of filters that have some interesting theoretical and numerical properties [8], which might be a more optimal choice for application to $P_{N}$ transport. Moreover, McClarren \& Hauck 31 presented results only for the $2 \mathrm{D}$ case, leaving open the question of how well their filtering approach performs in 3D. Also, this filtering scheme, as realized by [31, and as we will discuss in detail later in this paper, does not have a clear continuum limit as the spatial resolution and/or timestep approaches zero, which implies that the solution cannot be studied for spatial convergence.

In this paper, we reconsider the $P_{N}$ scheme and extend the work by McClarren \& Hauck 31] in a number of ways. Firstly, we reformulate the filtering procedure in such a way that it acquires a clear continuum limit. Secondly, we investigate a wider range of filter types and strength parameters. Finally, we perform calculations both in $2 \mathrm{D}$ and $3 \mathrm{D}$. For this we have we have developed the new radiation transport code Charon, whose ultimate goal is that of performing 3D, general relativistic multi-energy, multi-angle and velocity-dependent radiation-transport calculations. In this paper, we present the first step towards this goal.

Charon uses the semi-implicit scheme of McClarren et al. 30 for time integration. In this scheme, the streaming parts are treated explicitly with a second-order Runge-Kutta method, while the matter-coupling terms are treated implicitly because of the stiffness introduced by the coupling. Also, the implicit system of 30 is local to each spatial element, and for linearized matter-coupling terms this implicit integration scheme becomes trivial [30].

Although the timestep in the semi-implicit approach is limited by the Courant-Friedrichs-Lewy (CFL) condition based on the speed of light, this is not a serious drawback for the kind of applications that we have in mind. In radiation-hydrodynamics simulations, the timestep size would still be limited by the dependence of the matter properties (e.g., opacities and emissivities) on the matter temperature (and electron fraction, if we are dealing with neutrinos with conserved lepton number). Moreover, the semi-implicit approach is relatively easy to parallelize (e.g., via domain decomposition) and has lower communication requirements compared to fully implicit schemes. This should translate to significant advantages in massively-parallel large-scale radiation-hydrodynamics simulations. Moreover, in many relativistic systems, the sound speed of the fluid is comparable to the speed of light, and thus the radiation and hydrodynamics timescales are comparable, reducing the extra cost in an explicit treatment of the radiation streaming. The spatial discretization in Charon is based on the asymptotic-preserving linear discontinuous Galerkin (DG) scheme 28, 30.

This is the first in a series of three publications, with the present one dealing with static matter configurations and focusing exclusively on the transport methodology. For this reason, we do not consider the coupling with hydrodynamical equations. As a result, when an absorption occurs, the particles are simply removed from the system and do not increase the matter temperature. The second paper will include velocity-dependence and coupling to hydrodynamics, whereas in the subsequent publication we will present a fully general-relativistic algorithm.

The paper is organized as follows. In Section 2, we introduce the concept of radiation distribution function and the relativistic Boltzmann equation. In Section 3, we describe the numerical schemes used in our code for frequency (Section 3.1), angular (Section 3.2), spatial (Section 3.4), and time discretization (Section 3.5). In Section 4, we present numerical tests of these schemes. Finally, we summarize our results and provide our conclusions in Section 5. We use a spacetime signature $(-,+,+,+)$, with Greek indices running from 0 to 3 and the Latin indices from 1 to 3 . We also employ the standard convention for the 
summation over repeated indices.

\section{The relativistic Boltzmann equation}

\subsection{The distribution function for radiation}

Radiation is usually described in terms of the specific radiation intensity, $I$, defined such that

$$
\mathrm{d} \mathscr{E}=I \cos \theta \mathrm{d} A \mathrm{~d} \nu \mathrm{d} \Omega \mathrm{d} t,
$$

represents the energy of radiation in frequency range $\mathrm{d} \nu$ centered around $\nu$, traveling in direction $\Omega$ confined to a solid angle element $\mathrm{d} \Omega$, which crosses, within time interval $\mathrm{d} t$, an area $\mathrm{d} A$ oriented such that $\theta$ is the angle between the normal to the surface $\mathrm{d} A$ and direction $\Omega$ (e.g., [41). In the case of neutrino transport and/or in the relativistic case, it is more convenient to work directly with the distribution function, $F$, which gives the density of radiation carriers on a given point in phase space. The reason for this is that (1) the distribution function is a Lorentz-invariant quantity [35, and (2), as we discuss in more detail later, the distribution function allows us to compute the number density and the energy density of the radiation in a more natural way.

In order to introduce the distribution function, we first discuss the notion of single-particle phase space in special relativity. In this picture, the particles are described in terms of their positions in spacetime, $x^{\mu}$, and their momentum four-vector, $p^{\mu}$, as measured in a fiducial inertial frame. Using the normalization condition for timelike vectors, the four-momentum has only three independent components, which can be expressed in terms of three spatial components, $p^{i}$, or in terms of radiation frequency, $\nu$, and two angles $(\theta, \phi)$ that describe the propagation direction $2^{2}$

$$
p^{\mu}=\frac{h \nu}{c}(1, \cos \phi \sin \theta, \sin \phi \sin \theta, \cos \theta) .
$$

Since we wish to define the distribution function in terms of $p^{i}$, or, equivalently, in terms of $\nu, \phi$ and $\theta$, we need to construct a Lorentz-invariant volume element, $\mathrm{d} \Pi$, over the manifold of the allowed momentum four-vectors. This is accomplished with the choice 17

$$
\mathrm{d} \Pi=\frac{\mathrm{d} p^{1} \mathrm{~d} p^{2} \mathrm{~d} p^{3}}{-p_{0}}=\frac{h^{2} \nu}{c^{2}} \mathrm{~d} \nu \mathrm{d} \Omega .
$$

The distribution function is then defined in such a way that the quantity

$$
\mathrm{d} N=F p^{\mu} t_{\mu} \mathrm{d}^{3} x \mathrm{~d} \Pi=\frac{h^{3} \nu^{2}}{c^{2}} F \mathrm{~d}^{3} x \mathrm{~d} \nu \mathrm{d} \Omega,
$$

is the total number density of radiation carriers in a spatial volume element $\mathrm{d}^{3} x$ and phase-space volume element $\mathrm{d} \Pi$ with trajectories traversing a $t=$ const hypersurface with normal $\vec{t}=\partial_{t}$. Here, $t_{\mu}$ is the $\mu$ covariant component of the vector $\vec{t}=\left.\partial_{t}\right|^{3}$

Since $\mathrm{d}^{3} x=\mathrm{d} A \cos \theta \mathrm{d} t$ and the energy per particle is given by $h \nu$, we have $\mathrm{d} \mathscr{E}=h \nu \mathrm{d} N$. Using this and comparing equation (1) with (4), we obtain:

$$
I=\frac{h^{4} \nu^{3}}{c^{2}} F .
$$

Note that since $\mathrm{d} N$ is a scalar, $F$ is also a scalar quantity $\left.\right|^{4}$

${ }^{2}$ Assuming the radiation carriers to be massless, their energy and frequency are simply related as $\varepsilon=h \nu$.

${ }^{3}$ Note that $\mathrm{d}^{3} x$ is not a Lorentz-invariant quantity, while $p^{\mu} t_{\mu} \mathrm{d}^{3} x$ is one.

${ }^{4}$ Note that this relation slightly differs from the one frequently encountered in the neutrino-transport literature (e.g., 12 ):

$$
I=\frac{\varepsilon^{3}}{h^{3} c^{2}} g F=\frac{\nu^{3}}{c^{2}} g F,
$$




\subsubsection{The relativistic Boltzmann equation}

The special-relativistic Boltzmann equation can be written as 35

$$
p^{\mu} \frac{\partial F}{\partial x^{\mu}}=\mathbb{C}[F],
$$

where $\mathbb{C}$ is the collisional term describing the interaction of radiation with matter, while the left-hand-side of the equation describes the propagation of radiation. In order to compute $\mathbb{C}$, we express it in terms of the absorption, emission and scattering coefficients. To do that, we start by considering the evolution equation for the intensity of radiation 41 ,

$$
\frac{1}{c} \frac{\partial I}{\partial t}+n^{i} \frac{\partial I}{\partial x^{i}}=\eta-\kappa I+\frac{\kappa_{s}}{4 \pi} \int \frac{\nu}{\nu^{\prime}} K\left(\nu^{\prime}, \vec{n}^{\prime} \rightarrow \nu, \vec{n}\right) I\left(\nu^{\prime}, \vec{n}^{\prime}\right) \mathrm{d} \Omega^{\prime} \mathrm{d} \nu^{\prime},
$$

where $\eta$ represents the radiative emissivity of the matter, $\kappa$ is the total extinction coefficient and combines the absorption and scattering coefficients ${ }^{5} \kappa_{a}$ and $\kappa_{s}$, i.e., $\kappa=\kappa_{a}+\kappa_{s}$, and $K$ is the scattering kernel, expressing the probability of scattering from a given angle and frequency over to another angle and frequency [41].

Using equations (2), (5), and (7), it is easy to obtain an equation for $F$ in terms of the above extinction coefficients:

$$
p^{\mu} \frac{\partial F}{\partial x^{\mu}}=\frac{c^{2}}{h^{3}} \frac{\eta}{\nu^{2}}-h \nu \kappa F+\frac{h \nu \kappa_{s}}{4 \pi} \int\left(\frac{\nu^{\prime}}{\nu}\right)^{2} K\left(\vec{p}^{\prime} \rightarrow \vec{p}\right) F\left(\vec{p}^{\prime}\right) h \mathrm{~d} \nu^{\prime} \mathrm{d} \Omega^{\prime} .
$$

Notice that, since $F$ is a scalar, so is $\mathbb{C}[F]$, thus we find the classical result that $\eta / \nu^{2}$, and $\nu \kappa$ are invariant quantities 35].

Our scheme is in principle able to handle any type of scattering kernel, but for simplicity, here we will only consider the case of elastic scattering, e.g., scattering in which the radiation energy does not change. In this case, the scattering kernel can be expresses as [12],

$$
K\left(\nu^{\prime}, \vec{n}^{\prime} \rightarrow \nu, \vec{n}\right)=\frac{1}{4 \pi}\left[1+\sigma_{a} \vec{n} \cdot \vec{n}^{\prime}\right] \delta\left(\nu-\nu^{\prime}\right),
$$

where the scattering anisotropy is modeled using only one coefficient $\sigma_{a}$.

\section{Description of the scheme}

In general, the distribution function, $F$, is a function of 7 variables: the time and spatial coordinates, $x^{\mu}$, the frequency $\nu$ and the angles of propagation $\varphi$ and $\theta$. These variables are usually defined either in the Eulerian (inertial) frame or in the comoving frame (i.e., a set of frames, each of which has a velocity that instantaneously equals that of the matter element, e.g., 35, 26]). In the case of static matter, as the one considered in this paper, these two frames are identical. In the scheme implemented by the Charon code, the distribution function is expanded in the spatial coordinates using the linear DG basis and in the angular variables using spherical harmonics. The frequency is treated using the multi-group approach. This yields a large system of ordinary differential equations that is then evolved in time using a semi-implicit time integrator. The details of the discretization are discussed in this Section.

\footnotetext{
where $g$ is the statistical weight of the particles $(g=1$ for massless neutrinos, $g=2$ for photons) and $\varepsilon$ is the particle energy. This is due to three reasons: First, our specific intensity given by Eq. 11 is defined in terms of energy per frequency interval, in contrast to energy per energy interval, as used in the neutrino-transport literature. Moreover, our distribution function already contains the factor $g$, as can be seen from its relation to the total number density of radiation carriers given by Eq. 4 . Finally we use the Lorentz-invariant volume element given by Eq. $\sqrt{3}$ instead of $\mathrm{d}^{3} p$.

${ }^{5}$ Hereafter, the absorption or scattering extinction coefficients are defined as absorption or scattering opacitites or inverse mean-free paths.
} 


\subsection{Frequency discretization}

We consider the case in which the distribution function has compact support in a frequency space given by the interval $\left[0, \nu_{\max }\right]$. Although this is not strictly valid in the general case, radiation usually has negligible contribution above some cut-off frequency. Therefore, in many practical applications, one can choose $\nu_{\max }$ to be sufficiently large so that there is little radiation beyond this frequency. For simplicity of illustration, we introduce a uniform grid in this frequency space as $\nu_{n}=n \Delta \nu, n=0,1, \ldots, N_{\nu}+1$, where $\Delta \nu=\nu_{\max } /\left(N_{\nu}+1\right)$ (an extension to a non-equidistant grid is conceptually trivial). The associated intervals $\left[\nu_{n}, \nu_{n+1}\right]$ are commonly called frequency or energy groups. Using these groups, we can construct an orthonormal basis $\left\{\chi_{n}\right\}_{n=0}^{N_{\nu}}$ as

$$
\chi_{n}(\nu)=\left\{\begin{array}{ll}
1 / \sqrt{V_{n}}, & \text { if } \nu \in\left[\nu_{n}, \nu_{n+1}\right], \\
0, & \text { otherwise, }
\end{array} \quad V_{n}=\int_{\nu_{n}}^{\nu_{n+1}} h^{3} \nu^{2} \mathrm{~d} \nu=\frac{h^{3}}{3}\left(\nu_{n+1}^{3}-\nu_{n}^{3}\right) .\right.
$$

We then expand a function $f \in L^{1}\left(0, \nu_{\max }\right)$ on this basis as (for clarity we report the summation symbols in the expressions below)

$$
f_{N_{\nu}}(\nu)=\sum_{n=0}^{N_{\nu}} f^{n} \chi_{n}(\nu), \quad f^{n}=\frac{1}{\sqrt{V_{n}}} \int_{\nu_{n}}^{\nu_{n+1}} f(\nu) h^{3} \nu^{2} \mathrm{~d} \nu .
$$

The truncated expansion, $f_{N_{\nu}}$, is then a first-order accurate (in $L^{1}$-norm) approximation of $f$. We point out that, thanks to our choice of basis (10), the final expansion of the distribution function will involve integrals performed with respect to the volume element in (3). This allows us to ensure exact conservation of the number of radiation particles in the numerical treatment of the transport equation. Also, this choice of the basis can be easily generalized to the case of curved spacetimes (which will be the subject of our future work), where ensuring conservation of radiation particles is particularly involved [15].

\subsection{Angular discretization}

As orthonormal basis on the unit 2-sphere, $S_{1}$, we consider the real spherical harmonics, $Y_{\ell m}$ (see Appendix A, whose orthonormality conditions are

$$
\int_{S_{1}} Y_{\ell m}(\varphi, \theta) Y_{\ell^{\prime} m^{\prime}}(\varphi, \theta) \mathrm{d} \Omega=\delta_{m m^{\prime}} \delta_{\ell \ell^{\prime}} .
$$

As a result, any function $f \in L^{2}\left(S_{1}\right)$ can be expanded in spherical harmonics as

$$
f_{N}(\varphi, \theta)=\sum_{\ell=0}^{N} \sum_{m=-\ell}^{\ell} f^{\ell m} Y_{\ell m}(\varphi, \theta), \quad \quad f^{\ell m}=\int_{S_{1}} f(\varphi, \theta) Y^{\ell m}(\varphi, \theta) \mathrm{d} \Omega,
$$

where we have used the notation $Y^{\ell m}$ to denote the complex conjugate of $Y_{\ell m}$ (which is equal to $Y_{\ell m}$ since we are working with real spherical harmonics). If $f$ is a smooth function, $f_{N}$ will converge to $f$ with spectral accuracy in the $L^{2}-$ norm 8 .

\subsection{The multi-group $P_{N}$ scheme}

We consider the following ansatz for the expansion of the distribution function:

$$
F\left(x^{\alpha}, \nu, \varphi, \theta\right)=\sum_{n=0}^{N_{\nu}} \sum_{\ell=0}^{N} \sum_{m=-\ell}^{\ell} F^{n \ell m}\left(x^{\alpha}\right) \chi_{n}(\nu) Y_{\ell m}(\varphi, \theta),
$$

where

$$
F_{n \ell m}\left(x^{\alpha}\right)=\int_{0}^{\infty} h^{3} \nu^{2} \mathrm{~d} \nu \int_{\mathcal{S}_{1}} \mathrm{~d} \Omega F\left(x^{\alpha}, \nu, \varphi, \theta\right) Y_{\ell m}(\varphi, \theta) \chi_{n}(\nu)
$$


To simplify the notation, we introduce the multi-index $A=\{n, \ell, m\}$, and the basis functions,

$$
\Psi_{A}(\nu, \varphi, \theta) \equiv \chi_{n}(\nu) Y_{\ell m}(\varphi, \theta),
$$

so that Eq. 13 becomes

$$
F\left(x^{\alpha}, \epsilon, \varphi, \theta\right)=\sum_{A} F^{A}\left(x^{\alpha}\right) \Psi_{A}(\epsilon, \varphi, \theta)=F^{A} \Psi_{A} .
$$

Note that the space spanned by our basis $\left\{\Psi_{A}\right\}$ is a vector space. We adopt the usual convention of denoting vector components with an upper index and co-vector components with a lower index. Linear operators acting on vectors and co-vectors will have upper and lower indices associated with their decomposition on an appropriate tensor product combination of the canonical basis $\left\{\Psi_{A}\right\}$ and its dual $\left\{\Psi^{A}\right\}$, defined by the requirement that $\int \Psi^{A} \Psi_{B} p^{0} \mathrm{~d} \Pi=\delta^{A}{ }_{B}$. Note that, thanks to the orthornormality of real spherical harmonics $\Psi^{A}=\Psi_{A}$.

Inserting Eq. (14) into Eq. (6), we obtain:

$$
p^{0} \frac{\partial F^{B}}{\partial t} \Psi_{B}+p^{k} \frac{\partial F^{B}}{\partial x^{k}} \Psi_{B}=\mathbb{C}[F] .
$$

Multiplying Eq. 15 by $\Psi^{A}$ and integrating with respect to dח, we then obtain

$$
\frac{\partial F^{A}}{\partial t}+\mathcal{P}^{k A} \frac{\partial F^{B}}{\partial x^{k}}=\mathbb{S}^{A}[F]
$$

where we have again used Eq. (2) and exploited the orthonormality of the basis. We have also defined

$$
\mathcal{P}^{k A}{ }_{B} \equiv \int p^{k} \Psi^{A} \Psi_{B} \mathrm{~d} \Pi,
$$

and

$$
\mathbb{S}^{A}[F] \equiv \int \mathbb{C}[F] \Psi^{A} \mathrm{~d} \Pi .
$$

The coefficients (17) can be computed exactly using a quadrature formula of high-enough order (see Appendix A. Since they are independent of position and time, they can be pre-computed and stored for later usage.

The spectral decomposition of $\mathcal{P}^{k A}{ }_{B}$, which determines the behavior of Eq. $[16$, is well known, see e.g. 11]. In particular, it has been shown that the eigenvalues are strictly bounded by the speed of light c. While this implies that there is no superluminal propagation of radiation, it leads to slower-than-light motion of radiation waves for finite $N$ (the radiation velocity converges to the correct value with increasing $N)$ [10. This is particularly evident in low-order $P_{N}$ free-streaming solutions. For instance, the maximum propagation speed for $P_{1}$ is $c / \sqrt{3}$. Filtering can also affect the propagation velocity of radiation [31. In the multidimensional case, $\mathcal{P}^{k A}{ }_{B}$ has also zero-speed modes that have to be treated carefully in Godunov-based schemes to avoid numerical instabilities [11.

In the general case, the source term (18) has to be computed at run-time, but for the particular case of a source term in the form (9), and assuming that the opacity coefficients are constant in each of the energy groups (as commonly done in multi-group schemes [41]), the source terms can be pre-computed up to constant factors. Under these assumptions, the source term becomes

$$
\mathbb{S}^{A}[F]=e^{A}-\kappa_{n} F^{A}-\kappa_{s, n}\left[\perp^{A}{ }_{B}-\sigma_{a, n} \Delta^{A}{ }_{B}\right] F^{B}=e^{A}+S^{A}{ }_{B} F^{B},
$$

where

$$
e^{A} \equiv \int\left[\frac{c^{2} \eta}{h^{4} \nu^{3}}\right] \Psi^{A}(\nu, \varphi, \theta) h^{3} \nu^{2} \mathrm{~d} \nu \mathrm{d} \Omega
$$

\footnotetext{
${ }^{6}$ Notice that the term in square brackets is the number of emitted particles.
} 


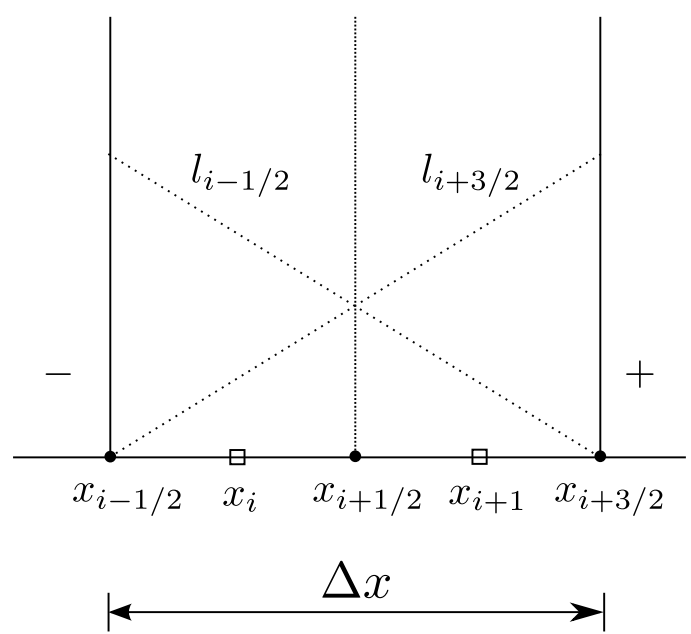

Figure 1: The grid structure for the spatial linear discontinuous Galerkin discretization. The white squares represent the cell centers, while the black dots show the cell interfaces. The dotted lines show the values of the Lagrangian basis $l_{i-1 / 2}, l_{i+3 / 2}$. Finally the + and - show the interfaces where the inter-element fluxes, $\mathcal{F}^{ \pm}$, see $[23]$, are computed.

$\perp{ }_{B}$ is the projector perpendicular to $Y_{00}$,

$$
\perp_{B}^{A} \equiv \delta_{B}^{A}-\delta^{A}{ }_{n 00} \delta^{n 00}{ }_{B},
$$

and $\Delta_{B}^{A}$ is the anisotropy matrix:

$$
\Delta_{B}^{A} \equiv \int h^{3} \nu^{2} \mathrm{~d} \nu \mathrm{d} \Omega \Psi^{A}(\nu, \varphi, \theta) \int \Psi_{B}\left(\nu, \varphi^{\prime}, \theta^{\prime}\right) \vec{n}^{\prime} \cdot \vec{n} \mathrm{~d} \Omega^{\prime}
$$

which can also be pre-computed. Note that we have denoted the opacity coefficients in the energy group $\left[\nu_{n}, \nu_{n+1}\right]$ with the subscript $n$.

\subsection{Spatial discretization}

We discretize the system of Eqs. (16) in space using the asymptotic-preserving (AP) linear DG scheme, see e.g. 28, 30. The scheme we present here is restricted to the case of orthogonal grids, but we point out that discontinuous Galerkin methods can be (and have been) extended to general, unstructured grids [19. We recall that a scheme is AP if it reproduces a discretization of the diffusion limit of the continuum transport equation in the limit of small mean-free-path. This is an important property since it guarantees that the diffusion of radiation has a correct rate even if the mean-free-path is small compared to the spatial grid size. If a scheme is not asymptotically preserving, then the diffusion rate becomes unphysical when the mean-free-path is unresolved.

For simplicity of notation, we consider a simplified 1D version of Eq. (16):

$$
\frac{\partial F^{A}}{\partial t}+\mathcal{P}^{1 A} \frac{\partial F^{B}}{\partial x}=0
$$

where the multidimensional case will be discussed at the end of this Section. Furthermore, we employ a uniform numerical grid $x_{i}=i \delta x$, while extension to a non-uniform grid is conceptually trivial. We then choose the points of the grid that are used to construct elements of width $\Delta x=2 \delta x$, as shown in Fig. 1 . This rather uncommon grid structure has been adopted to ease the integration of Charon with existing general-relativistic hydrodynamic codes that use traditional finite-volume schemes.

In the classical linear DG scheme, the degrees of freedom are usually identified with the one-sided limits of the solution at the points $x_{i-1 / 2}$ and $x_{i+3 / 2}$ from the interior of the element, while in our case we evolve 
the cell-centered values at $x_{i}$ and $x_{i+1}$. The semi-discrete equations for the evolution of these cell-centered values can be easily obtained, since any function $u$ in the finite-element space can be written as

$$
u(x)=u_{i-1 / 2} l_{i-1 / 2}(x)+u_{i+3 / 2} l_{i+3 / 2}(x),
$$

where

$$
l_{i-1 / 2}(x)=1-\frac{x-x_{i-1 / 2}}{x_{i+3 / 2}-x_{i-1 / 2}}, \quad \quad l_{i+3 / 2}(x)=\frac{x-x_{i-1 / 2}}{x_{i+3 / 2}-x_{i-1 / 2}}
$$

so that

$$
u_{i-1 / 2}=\frac{3}{2} u_{i}-\frac{1}{2} u_{i+1}, \quad u_{i+3 / 2}=-\frac{1}{2} u_{i}+\frac{3}{2} u_{i+1}
$$

and

$$
u_{i}=\frac{3}{4} u_{i-1 / 2}+\frac{1}{4} u_{i+3 / 2}, \quad u_{i+1}=\frac{1}{4} u_{i-1 / 2}+\frac{3}{4} u_{i+3 / 2} .
$$

The resulting numerical scheme is

$$
\Delta x \frac{\mathrm{d} F_{i}^{A}}{\mathrm{~d} t}=\mathbb{F}^{A}{ }_{i}
$$

where the flux terms in the element $\left[x_{i-1 / 2}, x_{i+3 / 2}\right]$ are given by

Here,

$$
\mathbb{F}^{A}{ }_{i} \equiv \frac{3}{2} \mathcal{F}^{-}-\overline{\mathcal{F}}-\frac{1}{2} \mathcal{F}^{+}, \quad \quad \mathbb{F}^{A}{ }_{i+1} \equiv \frac{1}{2} \mathcal{F}^{-}+\overline{\mathcal{F}}-\frac{3}{2} \mathcal{F}^{+} .
$$

is the average flux, while

$$
\overline{\mathcal{F}} \equiv \frac{1}{2}\left[\left(\mathcal{P}^{1 A}\right)_{i} F_{i}^{B}+\left(\mathcal{P}^{1 A}{ }_{B}\right)_{i+1} F_{i+1}^{B}\right]
$$

$$
\mathcal{F}^{-} \equiv \frac{1}{2}\left[\mathcal{P}^{1 A}{ }_{B}\left(F^{B}{ }_{L}+F^{B}{ }_{R}\right)-\mathcal{R}^{1 A}{ }_{C} \max \left(v,\left|\Lambda^{1 C}{ }_{D}\right|\right) \mathcal{L}^{1 D}{ }_{B}\left(F^{B}{ }_{R}-F^{B}{ }_{L}\right)\right],
$$

is the flux computed from the exact solution of the Riemann problem at $x_{i-1 / 2}$ with the left " $L$ " and right " $R$ " states $F^{A}{ }_{L}$ and $F_{R}^{A}$. The term $\mathcal{F}^{+}$is the flux across $x_{i+3 / 2}$ and is calculated analogously to $\mathcal{F}^{-}$. In the previous equations, we have decomposed $\mathcal{P}^{1 A}{ }_{B}$ as

$$
\mathcal{P}^{1 A}{ }_{B}=\mathcal{R}^{1 A}{ }_{C} \Lambda^{1 C}{ }_{D} \mathcal{L}^{1 D}{ }_{B},
$$

where $\mathcal{R}^{1 A}{ }_{C}$ are the matrices of the right eigenvectors, while $\Lambda^{1 C}{ }_{D}$ and $\mathcal{L}^{1 D}{ }_{B}$ are the eigenvalues and the left eigenvectors of $\mathcal{P}^{1 A}{ }_{B}$, respectively. We have also introduced $v>0$ which is taken to be the first positive abscissa of the adopted Legendre quadrature and it is used to introduce extra numerical dissipation on the zero speed modes similarly to what is done in [11. Notice again that the spectral decomposition of $\mathcal{P}^{1 A}{ }_{B}$, discussed in the previous Section, can also be pre-computed at the beginning of the calculations.

Having described the numerical scheme for the 1D problem, we can construct the multidimensional numerical scheme for Eq. (16) by repeating the same construction in every direction:

$$
\frac{\mathrm{d} F^{A}{ }_{i, j, k}}{\mathrm{~d} t}=e^{A}+S^{A}{ }_{B} F^{B}+\frac{1}{\Delta x} \mathbb{F}^{A}{ }_{i, j, k}+\frac{1}{\Delta y} \mathbb{G}^{A}{ }_{i, j, k}+\frac{1}{\Delta z} \mathbb{H}^{A}{ }_{i, j, k},
$$

where the fluxes in the $y$ and $z$ direction, $\mathbb{G}$ and $\mathbb{H}$, are computed analogously to the ones in the $x$ direction.

To avoid creation of false extrema in the numerical solution we use the slope limiting technique 19. Among the different limiters that we have implemented are (1) the so-called "step limiter", which simply reduces the scheme to a first order discontinuous Galerkin scheme, (2) the "minmod" and (3) the asymptoticpreserving "minmod2" limiters 33. The reason for using these particular limiters is that they have been well tested in the context of the transport equation (e.g., 33). Furthermore, the minmod2 limiter has been studied in detail in the context of linear DG methods, where it has been shown that it does not affect smooth solutions away from local extrema [18, thus yielding a scheme with a very small dissipation. 


\subsection{Time discretization}

For the time integration, we use the predictor-corrector method proposed by McClarren et al. [30]. In this approach, the streaming terms that model the transport of radiation are treated explicitly, while the source terms responsible for interaction with matter are treated implicitly. The use of this particular time integrator is motivated by the fact that this yields a relatively inexpensive, stable and asymptotic-preserving scheme. As discussed in Section 1, the fact that the streaming terms are treated explicitly makes this scheme particularly easy to parallelize, while the associated CFL constraint is not particularly demanding for applications involving fluid moving at relativistic velocities and general-relativistic gravity.

In order to simplify the notation, we rewrite Eq. (24) as

$$
\frac{\mathrm{d} F^{A}}{\mathrm{~d} t}=e^{A}+S^{A}{ }_{B} F^{B}+\mathcal{A}^{A}[F]
$$

where $\mathcal{A}^{A}[F]$ is a shorthand for the treatment of the spatial flux terms. For the time integration of Eq. 25), we use the following two-step semi-implicit asymptotic-preserving scheme. Given the solution $F_{k}^{A}$ at time $k \Delta t$, we first perform a predictor step

$$
\frac{F_{k+1 / 2}^{A}-F_{k}^{A}}{\Delta t / 2}=\mathcal{A}^{A}\left[F_{k}\right]+e_{k}^{A}+S_{B}^{A} F_{k+1 / 2}^{B},
$$

to obtain the solution at time $(k+1 / 2) \Delta t$ and then a corrector step:

$$
\frac{F_{k+1}^{A}-F_{k}^{A}}{\Delta t}=\mathcal{A}^{A}\left[F_{k+1 / 2}\right]+e_{k+1 / 2}^{A}+S_{B}^{A} F_{k+1}^{B},
$$

to obtain the solution at time $(k+1) \Delta t$. At both stages, the absorption, emission and scattering terms are treated implicitly, while the streaming terms are treated explicitly. The explicit part of this scheme is second-order accurate in time, while its implicit part is first-order accurate [30].

\subsection{Filtering}

Filtering is a common procedure to reduce the effects of the Gibbs phenomenon in spectral methods for numerical solution of partial differential equations [14, 42. Filtered spherical harmonics expansions have been successfully used in, e.g., meteorology (see e.g. 8] and references therein) and the effects of filtering on the truncation error of a spectral expansion are now reasonably well understood [48. However, the use of filters to mitigate (and, in most situations, remove) the occurrence of negative solutions in $P_{N}$ schemes has only been proposed recently by McClarren and Hauck 31.

In their work, the authors propose to filter the spherical harmonic expansion of the solution after each timestep using a spherical-spline filter. Applying this suggestion to the spherical harmonic expansion of $F$ we obtain (for clarity we report here the summation symbols)

$$
[\mathscr{F}(F)](\varphi, \theta)=\sum_{\ell=0}^{N} \sum_{m=-\ell}^{\ell}\left[\frac{1}{1+\alpha \ell^{2}\left(1+\ell^{2}\right)}\right] F^{\ell m} Y_{\ell m}(\varphi, \theta),
$$

where

$$
\alpha \equiv \frac{c \Delta t}{\Delta x} \frac{1}{N^{2}} \frac{1}{\left(\sigma_{t} L+N\right)^{2}},
$$

and $L$ is a characteristic length scale used to make $\alpha$ dimensionless, while $\sigma_{t}$ is chosen to be of the same order of magnitude as $\kappa$.

The filtered spherical harmonics, $F P_{N}$, scheme has several interesting properties. Filtering has been found to be very effective and robust in removing numerical oscillations in $P_{N}$ solutions, while preserving the rotational invariance of the scheme. Furthermore, for this particular choice of $\alpha$, filtering turns off automatically in the limit $N \rightarrow \infty$, thus it does not spoil the convergence of the spherical harmonics expansion. 
However, one important drawback, also remarked by McClarren and Hauck [33], is that the filtered $P_{N}$ scheme does not have a clear continuum limit as $\Delta x, \Delta t \rightarrow 0$. This is unfortunate because it implies that the filtered $P_{N}$ scheme, $F P_{N}$, cannot be interpreted as a system of partial differential equations. This in turn means that the quality of the $F P_{N}$ solution will depend on the spatial grid resolution in a way that is hard to predict. The ultimate and most important implication is that an $F P_{N}$ solution cannot be studied for spatial convergence.

To solve this problem, we propose a modification/generalization of the $F P_{N}$ scheme as follows. We introduce a strength parameter, $s \geq 0$, to be specified later, and construct the filtered expansion as

$$
[\mathscr{F}(F)](\varphi, \theta)=\sum_{\ell=0}^{N} \sum_{m=-\ell}^{\ell}\left[\sigma\left(\frac{\ell}{N+1}\right)\right]^{s} F^{\ell m} Y_{\ell m}(\varphi, \theta),
$$

where $\sigma(\eta)$ is a filter function of order $p$, that is, a function $\sigma \in C^{p}\left(\mathbb{R}^{+} ;[0,1]\right)$ such that ${ }^{7}$

$$
\sigma(0)=1, \quad \sigma^{(k)}(0)=0, \text { for } k=1,2, \ldots p-1 .
$$

Notice that, since the filter strength depends only on $\ell$, this does not destruct the rotational invariance of the scheme 8 Furthermore, as the order of the spherical harmonics, $N$, increases, the effect of filtering automatically decreases, so that the convergence of the scheme for $N \rightarrow \infty$ is retained. More specifically, for a filter of order $p$, we expect a convergence order of $\sim p-1$, as suggested by Vandeven's theorem for Fourier expansion [48, see also [25].

In our analysis we have considered two second-order and two fourth-order filters. The first one is the classical second-order Lanczos filter?.

$$
\sigma_{\text {Lanczos }}(\eta)=\frac{\sin \eta}{\eta}
$$

while the second and third choices are given by the ErfcLog filter [7],

$$
\sigma_{\operatorname{ErfcLog}}(\eta)=\frac{1}{2} \operatorname{Erfc}\left\{2 p^{1 / 2}\left(|\eta|-\frac{1}{2}\right) \sqrt{\frac{-\log \left[1-4(\eta-1 / 2)^{2}\right]}{4(\eta-1 / 2)^{2}}}\right\}
$$

of order $p=2,4$. Finally, the fourth filter is the fourth-order spherical-spline filter

$$
\sigma_{\text {SSpline }}(\eta)=\frac{1}{1+\eta^{4}}
$$

We point out that, with our definition, the spherical-spline filter 32 is very similar to the one used in 31], but is not exactly equivalent. The reason for using a slightly different filter form is that the filter of 31 is not compatible with the form of Eq. (28) as it cannot be written in terms of a function $\sigma(\cdot)$ of $\ell /(N+1)$.

In addition, we have considered only even-order filters since the truncation error associated with these filters can be interpreted as a numerical viscosity of order higher than two [34, while for odd-order filters the leading truncation error is of the dispersion type 34. Moreover, we also do not consider filters of orders higher than 4. This is because, as we will see later, the fourth-order filters are already too weak to completely remove oscillations, suggesting that even higher order will be even less efficient.

\footnotetext{
7 Here we ignore the technical requirement for Vandeven's theorem that $\sigma^{(k)}(1)=0$ for $k=0,1,2, \ldots p-1$, which is not satisfied by our filters (nor by the one proposed by 31). This is a condition that does not influence the formal accuracy of the filtered expansion with respect to the unfiltered truncated expansion, but it is mainly needed to prove the convergence of the filtered expansion in the case in which the unfiltered expansion is not converging point-wise (for instance due to the presence of discontinuities) 24, 47].

${ }^{8}$ This is a consequence of the classical addition theorem for spherical harmonics (e.g., 7]).

${ }^{9}$ Note that the Lanczos filter is usually defined as $\sigma(\eta)=\sin \pi \eta / \pi \eta$ to have a first-order zero at $\eta=1$, as discussed in footnote 7 Our modified Lanczos filter yields a more uniform damping of high-order modes and works very well in our experiments.
} 
In our scheme, we filter the solution after each sub-step of the time integrator. This yields the following scheme

$$
\begin{aligned}
\frac{F_{*}^{A}-F_{k}^{A}}{\Delta t / 2} & =\mathcal{A}^{A}\left[F_{k}\right]+e_{k}^{A}+S^{A}{ }_{B} F^{B}{ }_{k+1 / 2}, \\
F_{k+1 / 2}^{A} & =\mathscr{F}^{A}{ }_{B} F^{B}{ }_{*}, \\
\frac{F^{A}{ }_{* *}-F_{k}^{A}}{\Delta t} & =\mathcal{A}^{A}\left[F_{k+1 / 2}\right]+e^{A}{ }_{k+1 / 2}+S^{A}{ }_{B} F^{B}{ }_{k+1}, \\
F_{k+1}^{A} & =\mathscr{F}_{B}^{A}{ }_{B} F^{B}{ }_{* *},
\end{aligned}
$$

where $\mathscr{F}_{B}^{A}$ is a diagonal matrix representing the filtering operation.

We should remark that both our scheme and the one by 31, cannot be interpreted as a continuum problem, in the sense that the equations $(33)-(36)$ do not, in general, represent a discretization of any system of partial differential equations. The main reason is that $\mathscr{F}^{A}{ }_{B}$ is not idempotent, i.e., $\mathscr{F}_{C}^{A} \mathscr{F}^{C}{ }_{B} \neq \delta^{A}{ }_{B}$, so that the filtering operations in Eqs. (34) and (36) do not have a well-defined behavior in the limit $\Delta t \rightarrow 0$. In the case in which $\mathscr{F}_{B}^{A}$ is idempotent, the scheme has indeed a continuum limit, but it can be easily demonstrated that the $F P_{N}$ method is just the $P_{M}$ method for some $M \leq N 10$

This problem can be solved by making the strength parameter $s$ (and $\mathscr{F}_{B}^{A}$ with it) depend on the timestep. In order to see that, we consider the behavior of our scheme for a given mode $u=F^{\ell m}$ with $\ell \neq 0$. Let $q=\sigma(\ell /(N+1))$, where $\sigma$ is a filter function. Then the effect of filtering on $u$ in each of the two filtering steps in, e.g., Eq. (36) is simply:

$$
u_{k+1}=q^{s} u_{* *}
$$

This can be rewritten as

$$
\frac{u_{k+1}-u_{* *}}{\Delta t / 2}=\frac{1}{\Delta t / 2}\left[q^{s}-1\right] u_{* *}
$$

If we let $s=\beta \Delta t$, then, in the limit of $\Delta t \rightarrow 0$, we obtain

$$
\frac{\mathrm{d} u}{\mathrm{~d} t}=\beta \log q u .
$$

In other words, we can interpret filtering as a first-order, operator split, discretization of the system of equations

$$
\frac{\partial F^{A}}{\partial t}+\mathcal{P}^{k A}{ }_{B} \frac{\partial F^{B}}{\partial x^{k}}=e^{A}+S_{B}^{A} F^{B}+\beta L^{A}{ }_{B} F^{B}
$$

where $L_{B}^{A}$ is a diagonal matrix with coefficients $\log \sigma(\ell /(N+1))$. This is the desired continuum limit. The physical interpretation is that filtering is equivalent to a forward-peaked scattering operator (notice that $\sigma(0)=1)$. Finally, we note that we can estimate the filter effective opacity by looking at the dissipation rate for the highest-order multiple of the expansion as

$$
\sigma_{\mathrm{eff}}=-\beta \log \sigma(N /(N+1))
$$

\section{Tests}

In this Section, we present some tests of the numerical schemes described above as implemented in our Charon code. Charon uses 3D Cartesian coordinates in space and is currently parallelized employing hybrid OpenMP/MPI parallelization using the domain decomposition method. It uses the open-source Cactus Computational Toolkit [23, 1], which provides MPI parallelization, input/output, and restart capabilities.

\footnotetext{
${ }^{10}$ The reason is that the only idempotent filter is the cut-off filter, that is, the filter that simply sets to zero all the modes with $\ell>M$ for some $M$, while leaving unaffected all the modes with $\ell \leq M$.
} 


\section{1. $1 D$ diffusion of a step function}

In this first test, we primarily focus on verifying the ability of our scheme to handle diffusion of radiation when the opacity is high and the mean-free-path is small compared to the grid spacing. In this limit, the continuous hyperbolic transport equation displays parabolic character to leading order [33. Despite this, there is no guarantee that a numerical scheme for solving the hyperbolic system will be AP, that is, will reproduce a valid discretization of the asymptotic limit of the continuous equations (cf. the discussion in Section 3.4).

Consider therefore the following initial conditions for the radiation energy density $E \equiv \int I \mathrm{~d} \Omega \mathrm{d} \nu$, in a non-moving infinite medium with a constant (isotropic and elastic) scattering opacity:

$$
E(z, t=0)=\mathrm{H}(z+1 / 2) \mathrm{H}(1 / 2-z),
$$

where $\mathrm{H}(\cdot)$ is the Heaviside step function. If the scattering opacity is high, the solution of the transport equation is well approximated by the solution of the diffusion equation. The corresponding diffusion equation has the following analytic solution to problem $(39)$

$$
E(z, t)=\frac{1}{2}\left[\operatorname{Erf}\left(\frac{z+1 / 2}{2 \sqrt{t / \tau}}\right)-\operatorname{Erf}\left(\frac{z-1 / 2}{2 \sqrt{t / \tau}}\right)\right],
$$

where $\operatorname{Erf}(\cdot)$ is the error function and $\tau \equiv 3 \kappa_{s} / c$ is the diffusion timescale, where $\kappa_{s}$ is the total scattering opacity, which we set $\kappa_{s}=10^{5}[$ e.g., 33 .

We employ five different schemes for this test: the step scheme (i.e., a DG scheme with step-limiter, or, equivalently a first-order FV scheme), two linear DG methods employing minmod and minmod2 limiters and two finite-volume (FV) methods also employing minmod and minmod2 limiters. In our implementation, the finite-volume scheme is obtained from the linear DG scheme by simply replacing the linear DG slope with the one obtained from the reconstruction procedure.

In all of our runs, we use the $P_{1}$ scheme because in 1D there are no negative solutions and thus filtering is not necessary, and because the radiation is nearly isotropic in such a diffusive regime so that $P_{1}$ scheme should be sufficiently accurate. We perform calculations using three different resolutions $\Delta z=0.16,0.08$ and $\Delta z=0.04$, with the grid ranging from -2 to 2 , and imposing periodic boundary conditions at the outer boundaries. We choose the CFL factor to be 0.25 and we recall that the maximum CFL factor that guarantees the $L^{2}$-stability of our scheme is $1 / 3$ in $1 \mathrm{D}[19$. In all of our tests, the CFL factor is mainly chosen for convenience in order to have a sufficient number of timesteps within a given time interval. Moreover, in many radiation-transport calculations, in the absence of hydrodynamical equations, the truncation error due to the time discretization is expected to be small compared to other sources of error (e.g., the angular and spatial discretization).

Figure 2 shows the radiation energy density as a function of $z$ coordinate for the run with $\Delta z=0.04$ at time $100 / c$. The thick black line corresponds to the analytic solution, while the other lines show numerical results obtained with the above schemes. The line with red circles corresponds to the linear DG with minmod2 limiter solution. The line with orange squares represents the solution from the finite-volume scheme with minmod2 reconstruction. Finally, the lines with blue diamonds and green triangles show the results obtained with linear DG with minmod and step limiters, respectively. Note that the different symbols also mark the value of the numerical solution at each grid point (i.e., we show two points for each element).

The linear DG method with minmod2 agrees well with the analytical result. This is expected since this scheme has the correct asymptotic limit 33. In contrast, all other schemes overestimate the diffusion rate. In particular, the step scheme produces the worst results. It reaches stationarity already at time $t / \tau \sim 10^{-4}$, which is much smaller than the diffusion timescale for this problem. The linear DG and finitevolume schemes with minmod yield identical results and for this reason we show only the results from the linear DG scheme. Both are only marginally better than the step algorithm. These results are in overall agreement with the ones reported by [33] for a very similar test.

The FV scheme with the minmod2 reconstruction produces results that are relatively accurate compared to the linear DG and finite-volume schemes with the minmod limiter, even though the observed diffusion 


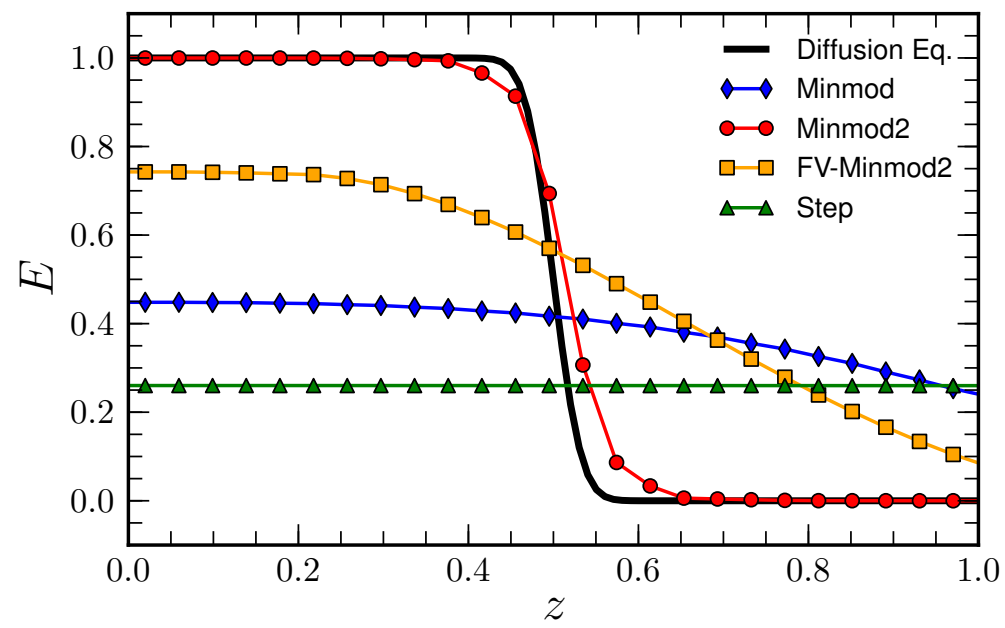

Figure 2: Radiation energy density as a function of the $z$ coordinate at time 100/c for the 1D diffusion of the step function $[39$. The thick black line represents the analytical solution of the corresponding diffusion equation, the line with red circles corresponds to the linear DG with minmod2 limiter solution, the line with orange squares represents the solution from the finite-volume scheme with minmod2 reconstruction, while the lines with blue diamonds and with green triangles show the results obtained with linear DG with minmod and step limiters, respectively. The symbols also mark the values of the numerical solution at each grid point (i.e., we show two points for each element).

timescale (at the current resolution) is still orders of magnitude larger than the physical one. Finally, we point out that the results obtained from the other lower-resolution runs (not shown here) are in overall agreement with the ones presented here.

\section{2. $1 D$ diffusion of a sine wave}

Next, we explore convergence of the numerical solution to the asymptotic one. To this scope we consider the 1D diffusion of a sine wave and thus adopt as initial conditions the energy density given by

$$
E(z, t=0)=3 \sqrt{4 \pi}\left[\sin \left(\frac{\pi z}{3}\right)+1\right]
$$

which has the following analytic solution in the diffusion limit

$$
E(z, t)=3 \sqrt{4 \pi}\left[1+\exp \left(-\frac{\pi^{2}}{9} \frac{t}{\tau}\right) \sin \left(\frac{\pi z}{3}\right)\right] .
$$

For this test, our computational domain ranges from -3 to 3 and we use eight different resolutions ranging from $\Delta z=0.3$ to $\Delta z=0.009375$. The CFL factor is chosen to be 0.3

Figure 3 shows the $L^{\infty}$-norm of the deviation of the numerical result from the asymptotic solution as a function of numerical resolution at time $t=1000 / c$. As expected, the linear DG scheme with minmod2 (line with red circles) exhibits approximately second-order convergence for the entire range of resolutions shown in the plot, while the linear DG with minmod (line with blue squares) starts converging only when $\Delta z \sim 10^{-1}$, afterwards it converges with order $\simeq 1.28$. The step scheme (line with green triangles) does not show any sign of convergence. These results are again consistent with what was observed in 33 . Finally, the finite-volume with minmod2 reconstruction (line with orange diamonds) exhibits second-order convergence even though this scheme is not asymptotic preserving.

\subsection{The 2D line-source problem}

As a first multidimensional problem used to benchmark different implementations of the filtered spherical harmonics discretization schemes we consider the so-called "line-source" problem, where we have initial 


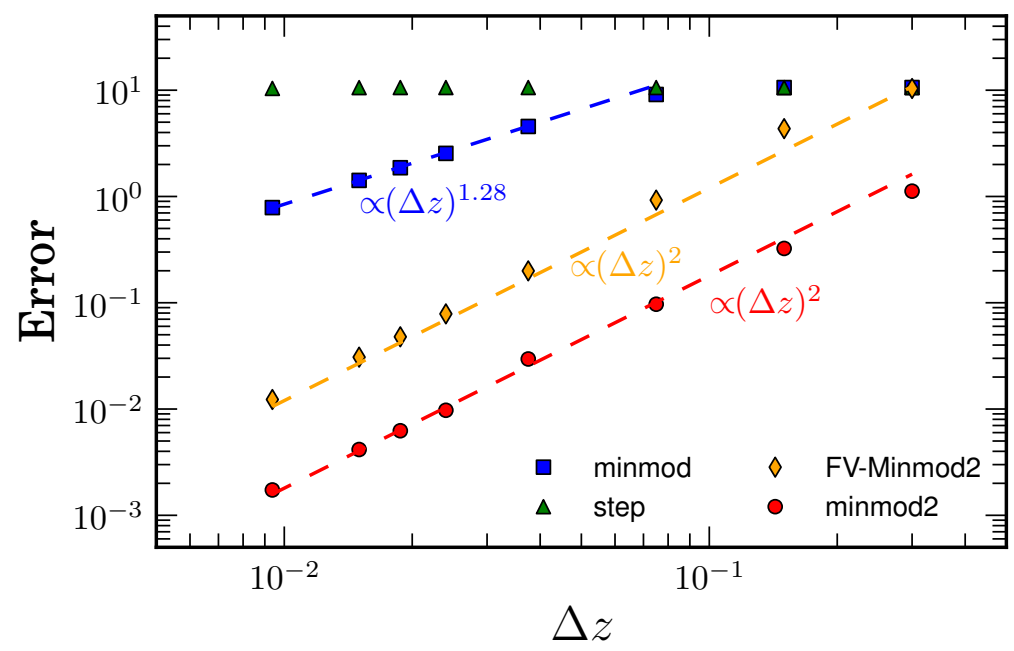

Figure 3: $L^{\infty}$-norm of the deviation of the numerical result from the asymptotic solution as a function of numerical resolution for the diffusion problem of a sine function. The lines with red circles and blue squares correspond to the linear DG schemes with minmod 2 and minmod limiters, respectively, while the line with orange diamonds represents the FV scheme with minmod 2 reconstruction. Finally, the line with green triangles corresponds to the step scheme.

conditions given by ${ }^{11}$

$$
I(x, y, z, \Omega, t=0)=\frac{E^{0}}{4 \pi} \delta(x) \delta(y),
$$

which represent an isotropic pulse of radiation with a total energy $E^{0}$ concentrated along the $z$-axis in vacuum [9]. This radiation field propagates in vacuum as it evolves in time according to the following analytical solution

$$
E(x, y, t)=\frac{E^{0}}{2 \pi} \frac{\mathrm{H}(c t-r)}{c t \sqrt{c^{2} t^{2}-r^{2}}},
$$

where $r \equiv \sqrt{x^{2}+y^{2}}$ is the distance from the $z$-axis and $\mathrm{H}(\cdot)$ is the unit step function and $\delta(\cdot)$ is the Dirac delta function. According to this solution, the radiation field consists of a front that forms a cylindrical shell that travels outwards at the speed of light, while in the interior, the radiation energy density smoothly decreases along the radial direction.

We point out that, while this test is actually one-dimensional in cylindrical coordinates, it becomes particularly challenging for radiation-transport codes, except for Monte-Carlo codes, when solved on a twodimensional Cartesian grid (as we do). In these coordinates, the radiation beam, which originates from a single spatial grid zone, has a very forward-peaked distribution in angle. This is a huge challenge for both spatial and angular discretization schemes. Moreover, such a configuration favors negative solutions in the $P_{N}$ scheme. Indeed, the analytical $P_{N}$ solution to this problem was shown to have regions with negative values of the energy [9, 32, 31, while the $P_{1}$ solution even exhibits a negative singularity [29]. For all the results presented here, we use a grid with resolution $\Delta x=\Delta y=0.02$ and a CFL factor of 0.0625 . Furthermore, we choose $E_{0}=\sqrt{4 \pi}$.

Figure 4 displays the colormap of the radiation energy density in the $x-y$ plane at $t=1 / c$. The upper left panel shows the analytic solution, while the upper right panel shows the pure $P_{7}$ solution (note the considerable difference in scale). As expected, the $P_{7}$ solution exhibits unphysical oscillations in the radial direction that are absent in the analytical solution to the full transport problem (44). The lower left panel of Fig. 4 shows instead the $F P_{7}$ solution with spherical-spline filter with effective opacity $\sigma_{\text {eff }}=20$ (the dependence of the solution on the order $N$ and on the value of the filter strength $\sigma_{\text {eff }}$ will be discusses below).

\footnotetext{
${ }^{11}$ The initial conditions are $3 \mathrm{D}$ but we exploit the cylindrical symmetry to solve the problem on the $(x, y)$ plane only.
} 


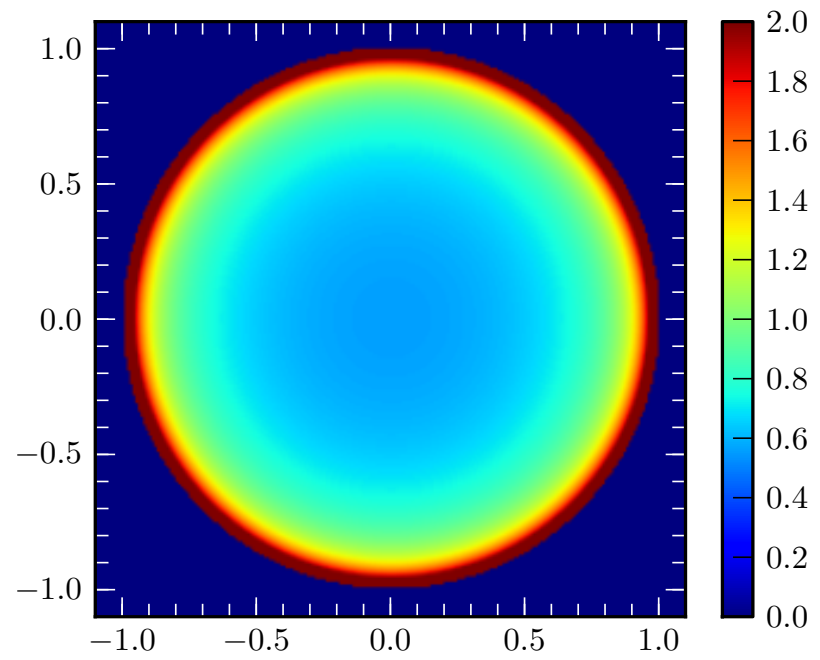

(a) Analytic solution

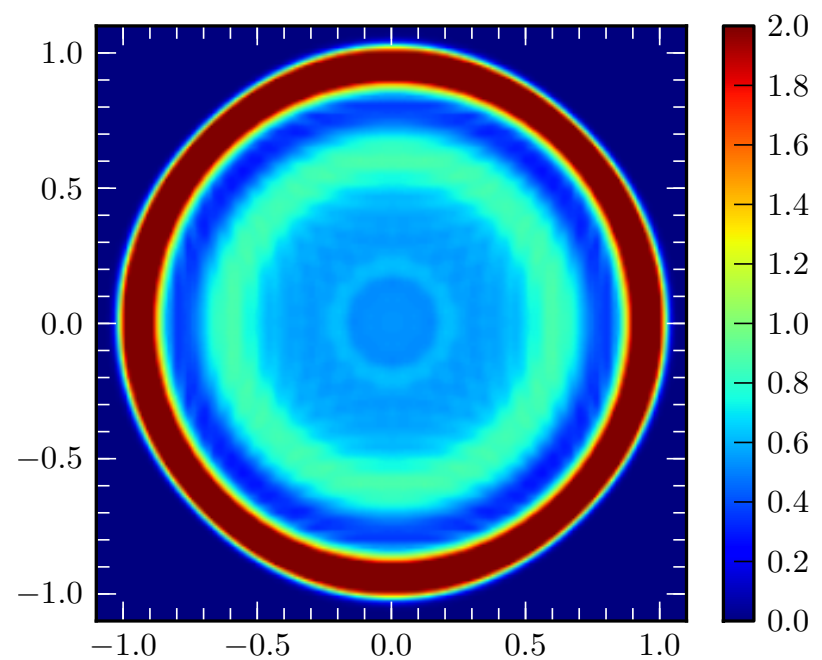

(c) $\mathrm{FP}_{7}$ with spherical-spline filter

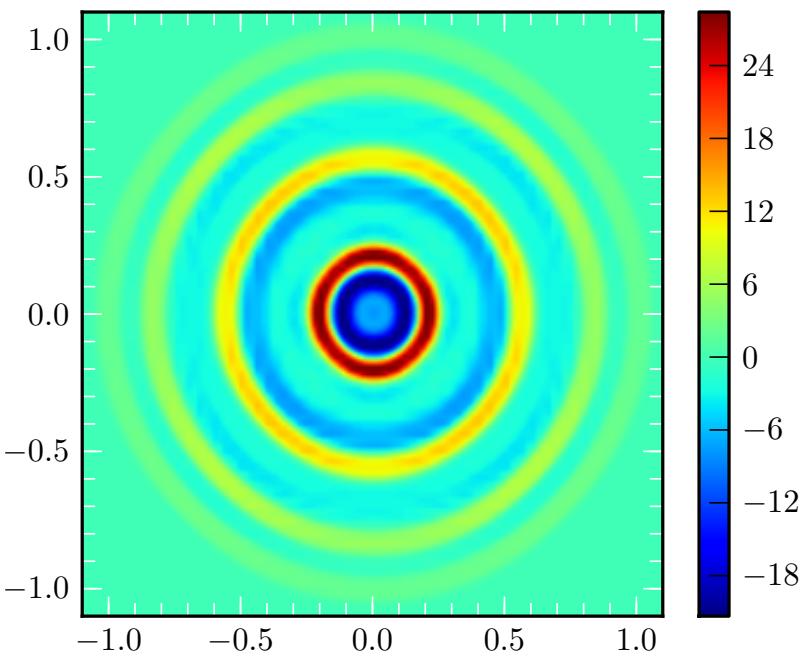

(b) Original $P_{7}$

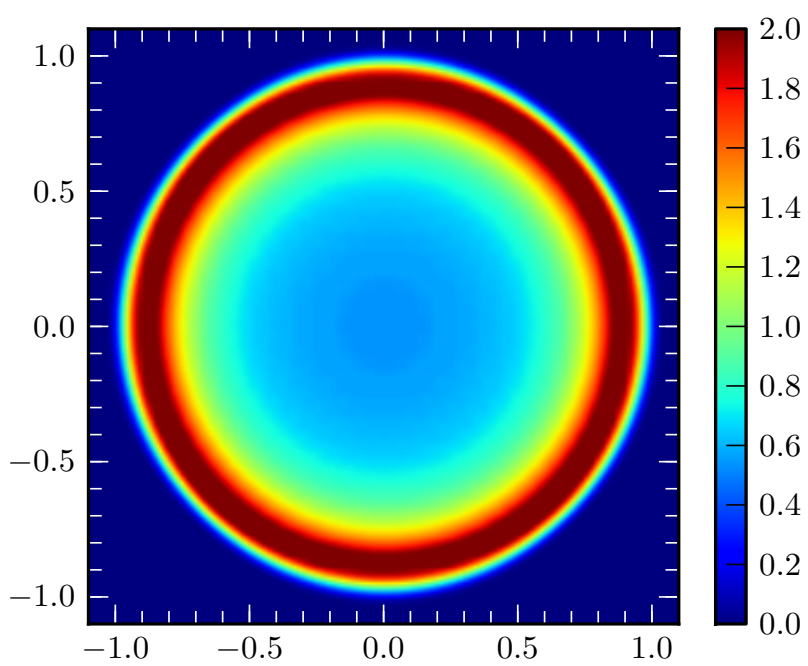

(d) $\mathrm{FP}_{7}$ with Lanczos filter

Figure 4: Colormaps of the radiation energy density on the $x-y$ plane at $t=1 / c$ for the line problem with different methods. The upper left panel shows the analytic solution, while the upper right panel shows the pure $P_{7}$ solution (note the considerable difference in scale). The lower left panel shows the $F P_{7}$ solution with spherical-spline filter with effective opacity $\sigma_{\text {eff }}=20$, while the lower right panel shows the $F P_{7}$ solution with the Lancsoz filter with the same effective opacity. 


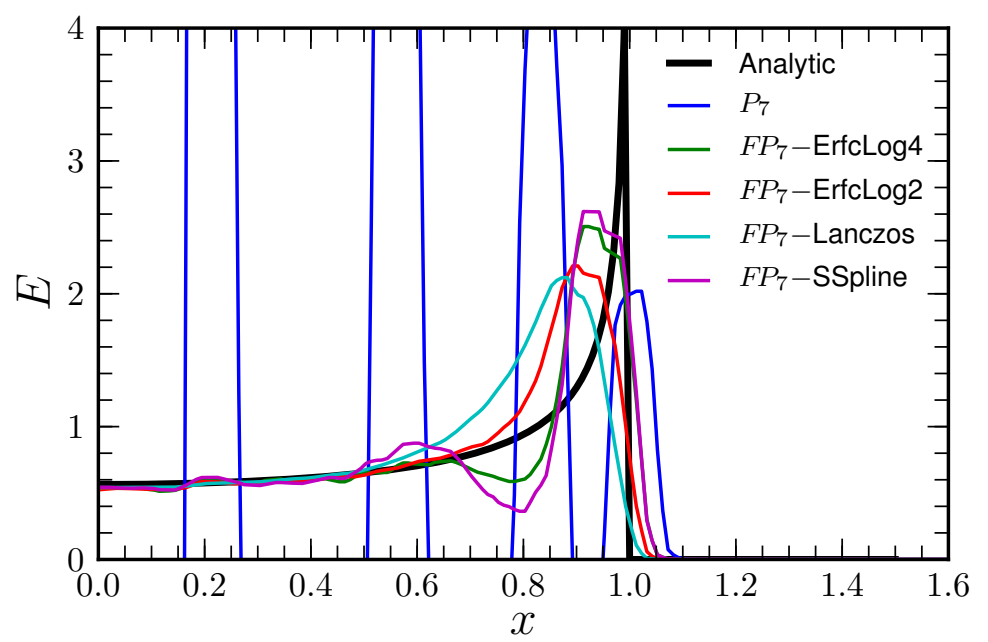

Figure 5: The radiation energy density as a function of the $x$ coordinate at $t=1 / c$ for the line problem test. The thick black line corresponds to the analytic solution, while the rest of the lines represent the results from $P_{7}$ calculations without filter (blue line), with fourth-order ErfcLog filter (green line), with the second-order ErfcLog filter (red line), with the Lanczos filter (cyan line), and with the fourth-order spherical-spline filter (magenta line).

In this case, the radial oscillations are significantly reduced compared to the unfiltered $P_{7}$ solution, similar to what was found in 31. Finally, the lower right panel shows the $F P_{7}$ solution with the Lancsoz filter with the same effective opacity. In this case, the amount of oscillations is even smaller and we get a result that is closer to the analytical one. The reason seems to be that the Lanczos filter, being a second-order filter, is more effective in reducing the appearance of oscillations. The solution obtained with the spherical-spline filter is still characterized by the presence of a ring structure that resembles the more oscillatory (unfiltered) $P_{N}$ solution. This structure does not disappear even for the values of the filter strength as high as $\sigma_{\text {eff }}=10^{4}$, suggesting that this is a result of a shortcoming of this particular filter. We point out that we have repeated these runs with the second-order (fourth-order) ErfcLog filter and we obtain a result similar to the one with the second-order Lanczos (fourth-order ErfcLog) filter, suggesting that the order of the filter plays the most important role, at least for this test.

A more quantitative measure of this test is shown in Fig. 5. where we plot a $1 \mathrm{D}$ cut of the radiation energy density as a function of the $x$-coordinate at $t=1 / c$. The thick black line again corresponds to the analytic solution, while the rest of the lines represent the results from $P_{7}$ calculations without filter (blue line), with the fourth-order ErfcLog filter (green line), with the second-order ErfcLog filter (red line), with the Lanczos filter (cyan line), and with the spherical-spline filter (violet line). All of these runs with filters are performed using a filter strength of $\sigma_{\text {eff }}=20$. We can easily notice again the presence of large oscillations in the unfiltered $P_{7}$ solution (which are larger than the scale of the plot). The fourth-order spherical-spline and ErfcLog filters are able to suppress most of the oscillations and remove the negative values. Nevertheless, both solutions are still affected by the oscillations (although to a much smaller extent compared to the $P_{7}$ solution). The second-order Lacnzos and ErfcLog filters, on the other hand, are able to remove most of the oscillations and give the best numerical solutions, overall.

The left panel of Fig. 6, which reports the radiation energy density as a function of the $x$ coordinate at $t=1 / c$, highlights how the quality of the solution varies with the (Lanczos) filter effective opacity. The black line again corresponds to the analytic solution, while the rest of the lines represent the $F P_{7}$ solution with the Lanczos filter of varying strength $\sigma_{\text {eff }}$. In the case of weak filters $\left(e . g ., \sigma_{\text {eff }}=1\right.$ or $\left.\sigma_{\text {eff }}=5\right)$, there are significant oscillations, whose amplitude is significantly reduced as we increase the filter strength. For example, for $\sigma_{\text {eff }}=20$, there are tiny oscillations, while for $\sigma_{\text {eff }}=50$ there are no noticeable oscillations. However, the quality of the solution with $\sigma_{\text {eff }}=50$ is actually worse than the one with $\sigma_{\text {eff }}=20$ (e.g., the radiation wavefront lags significantly behind the real solution) as a result of the large smearing of the 

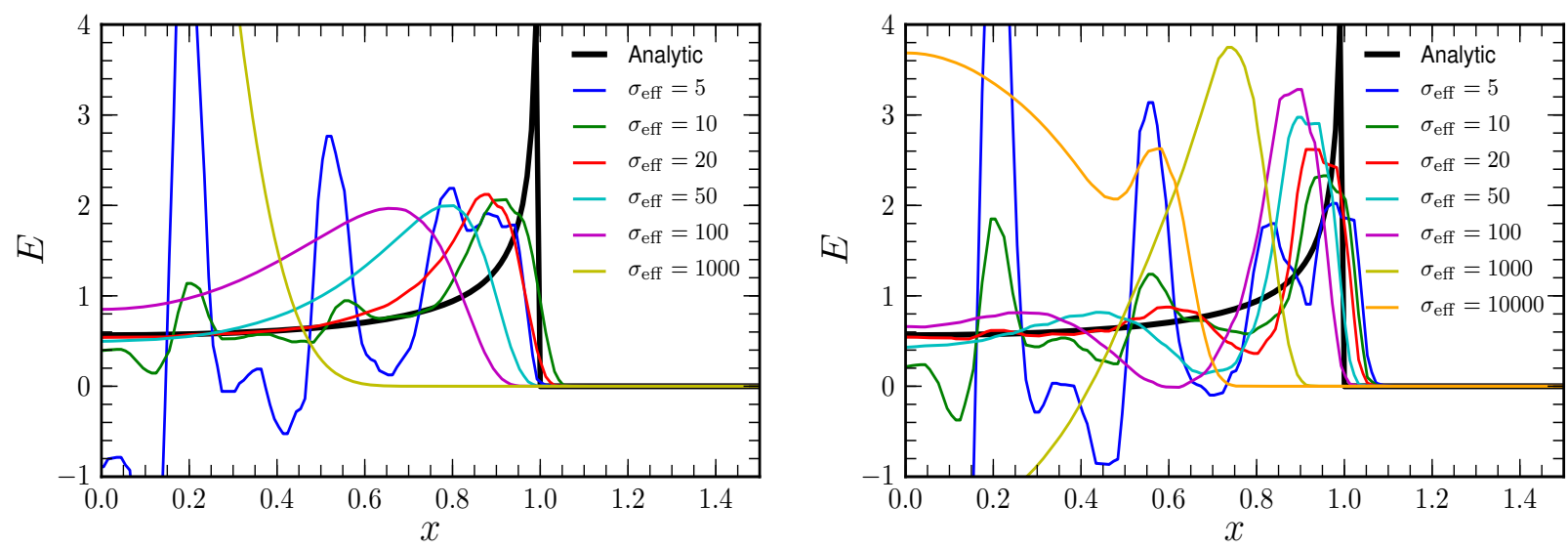

Figure 6: Left panel: radiation energy density as a function of the $x$ coordinate at $t=1 / c$ obtained from the $F P_{7}$ solution with the second-order Lanczos filter of varying strength $\sigma_{\text {eff }}$. The thick black line corresponds to the analytic solution, while the rest of the lines represent the numerical solutions corresponding to different $\sigma_{\text {eff }}$. Right panel: same as in the left panel but for the $\mathrm{FP}_{7}$ solution with the fourth-order spherical-spline filter of varying strength $\sigma_{\text {eff }}$.

radiation beam produced by the excessive filtering (this is even more evident for $\sigma_{\text {eff }}=100$ or $\sigma_{\text {eff }}=1000$ ). Therefore, the filter strength needs to be chosen large enough to damp oscillations and small enough to avoid excessive smearing of the solution. We have repeated these runs with the second-order ErfcLog filter and we again get similar results.

The left panel of Fig. 6 should be contrasted with the right one, where we show a study of the effect of different filter opacities for the same problem, but using the spherical-spline filter. The first thing to notice is that the spherical-spline filter is never able to completely remove the "ring" structure in the solution, even when the filter strength is so strong that the result resembles the solution of the diffusion equation for this problem. Secondly, the dependence of the filter behavior on the filter strength does not seem to be easily predictable: at first, as we increase filter strength, negative solution disappear (for $\sigma_{\text {eff }} \lesssim 100$ ), then they reappear for higher values of $\sigma_{\text {eff }}$ around 1000. We have repeated these runs with the fourth-order ErfcLog filter and we obtained similar results.

Finally, Fig. 7 shows the $F P_{N}$ solutions for $N=3,5,7,9$ and 11 with the Lanczos filter with $\sigma_{\text {eff }}=20$, and can be used to study the convergence of the $F P_{N}$ approximation to the analytic solution. In this plot, we can distinguish three different types of errors: (1) An error in the position of the radiation front, which is particularly evident for small $N$, is mainly related to the fact that the propagation speed of radiation is smaller than $c$ for low $N$. (2) An error in the profile of the radiation energy density behind the front, which is again particularly pronounced for small $N$, and is due to the fact that high angular resolution is needed to properly describe the very forward-peaked angular distribution of radiation. (3) A relatively large spreading of the radiation beam in space compared to the $x^{-1 / 2}, x=c t-r$, singularity in the analytical solution (44). This is an artifact of spatial discretization and mainly stems from the fact that the radiation beam originates from one spatial element and results in the presence of a "precursor" in the radiation front for high-order $F P_{N}$ (e.g., $F P_{11}$ ) solutions, where the spatial discretization scheme propagates the radiation front superluminally, despite the fact that the characteristic speeds of the $F P_{N}$ system are always smaller than $c$. Superluminal propagation of sharp features in numerical solutions of hyperbolic PDEs is a well known artifact of the spatial and temporal discretization of the equations. An in-depth explanation of this phenomenon for the case of the Maxwell equations can be found in 22. As we can see in the figure, the $F P_{N}$ solution nevertheless approaches the analytical one as we increase the order $N$ and, in particular, the errors associated with the angular discretization decrease to the point that the $F P_{11}$ solution yields only a small improvement with respect to the $F P_{9}$ one. In particular, a large contribution to the error in the $F P_{11}$ solution comes from the presence of the superluminal precursor discussed above. Since this precursor can only be attributed to the spatial discretization error, we can conclude that, at this particular resolution 


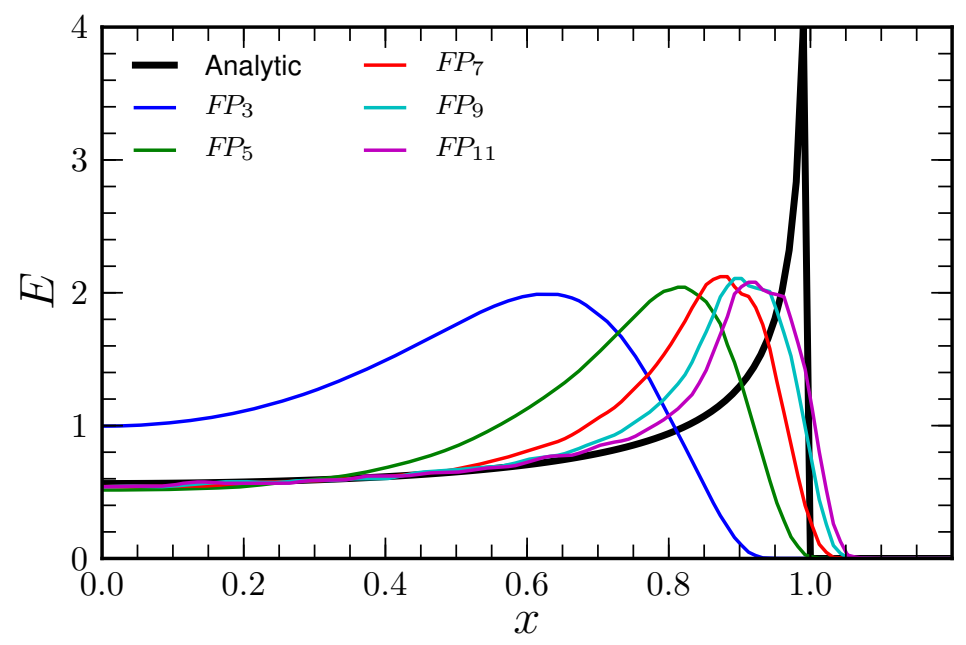

Figure 7: Radiation energy density as a function of the $x$ coordinate at $t=1 / c$ obtained from the $F P_{N}$ solution with the second-order Lanczos filter with $\sigma_{\text {eff }}=20$ for different values of order $N$. The thick black line represents that analytical solution, while the rest of the lines represent numerical solutions for different $N$. Clearly, the $F P_{N}$ solution approaches the analytical one as we increase the order $N$.

and order, the spatial discretization error is already comparable with the angular discretization ones. We have repeated these runs with the second-order ErfcLog filter and at half of the resolution and we again get similar results. These results show that our filtering strategy is able to recover the convergence of the $P_{N}$ scheme for this particular case, and that the second-order filters, unlike the fourth-order ones, do not require a delicate fine-tuning of the effective scattering opacity. In particular, $\sigma_{\text {eff }}$ can be chosen on the basis of the physics and geometry of the problem, in a way that is independent of the order of the employed $P_{N}$ scheme.

Overall, the results of this test confirm that the filtering approach is effective and robust in suppressing unphysical oscillations in the $P_{N}$ solution, even with moderately low-order $N$. Moreover, we find that the second-order Lancsoz and ErfcLog filters produce significantly better numerical results compared to the fourth-order spherical-spline and ErfcLog filters.

\subsection{A lattice problem}

Next, we consider another 2D problem consisting of a chessboard of highly scattering and highly absorbing square regions located around a central emitting square region. Although this geometry is not expected to be present in the astrophysical scenarios we are most interested in, it nevertheless represents a very demanding test of the capabilities of the different numerical schemes in complicated geometries.

In our calculation we use a setup illustrated in the upper left panel of Fig. 8, which consists of a central emitting square (shown in white) and 11 absorbing squares (shown in black) with a constant absorption opacity $\kappa_{a}=10$ surrounding the central emitting square. The space between the squares (shown in gray) and the central emitting region have a small uniform scattering opacity of $\kappa_{s}=1$. Each square has a linear size of 1 . The size of the computational domain is 7 along both axes. We choose a spatial resolution of $\Delta x=\Delta z=0.035$ and the CFL factor was set to $\approx 0.14$, with outgoing boundary conditions imposed at the outer boundary.

The remaining panels in Fig. 8 show the $\log _{10}$ of the radiation energy density produced by different schemes at a time $t=3.2 / c$, which roughly corresponds to the moment when the radiation reaches the outer boundary of the computational domain. The upper right panel corresponds to the $P_{7}$ solution. Not surprisingly, this solution has regions of negative energy density (shown in white), although the negative values reach at most a relatively small magnitude of $\sim 10^{-5}$. All other solutions are computed with the Lanczos filter with effective opacity $\sigma_{\text {eff }}=5$. We find that $\sigma_{\text {eff }} \gtrsim 5$ is necessary to avoid the appearance of negative solutions. The middle-left panel represents the $F P_{1}$ solution, which does not have negative regions, 


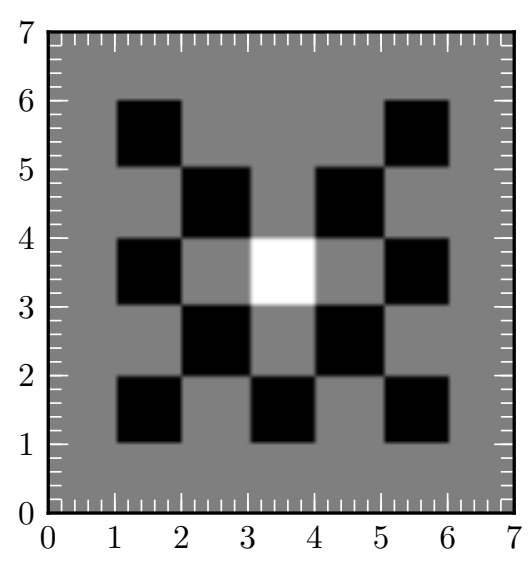

(a) Grid

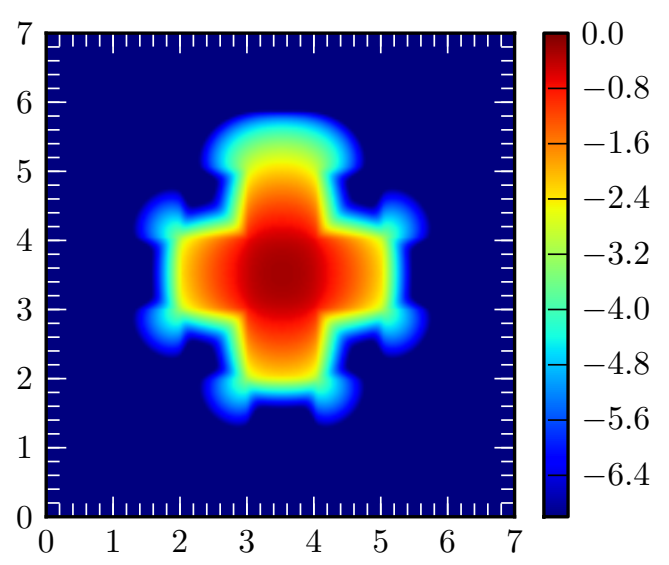

(c) $F P_{1}$ solution

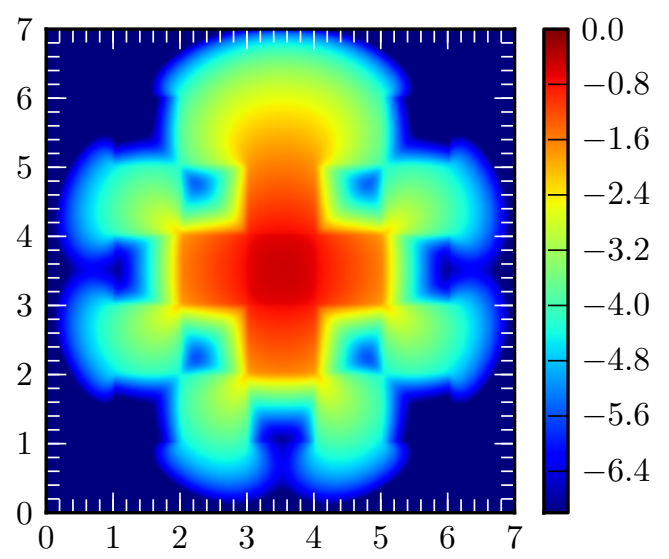

(e) $F P_{5}$ solution

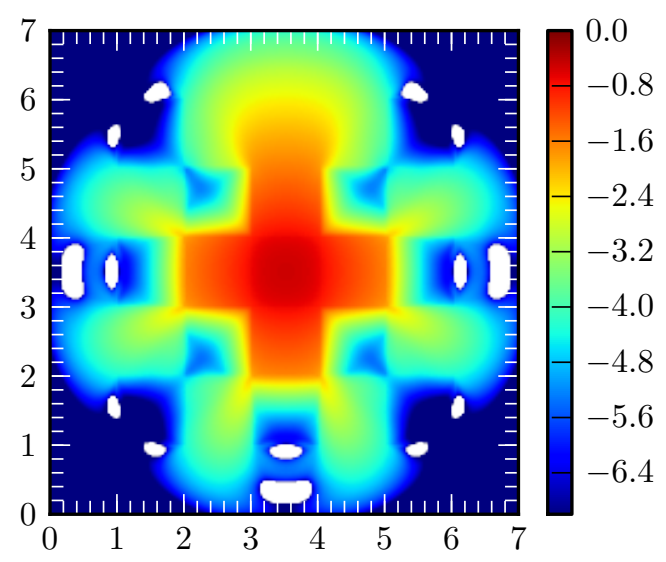

(b) $P_{7}$ solution

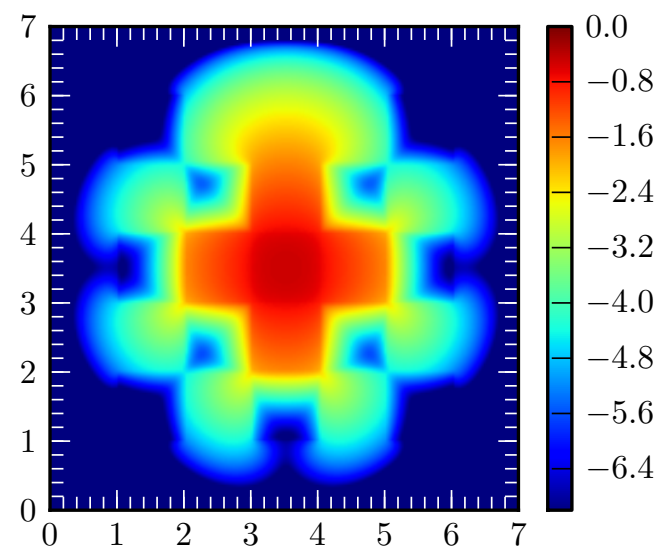

(d) $\mathrm{FP}_{3}$ solution

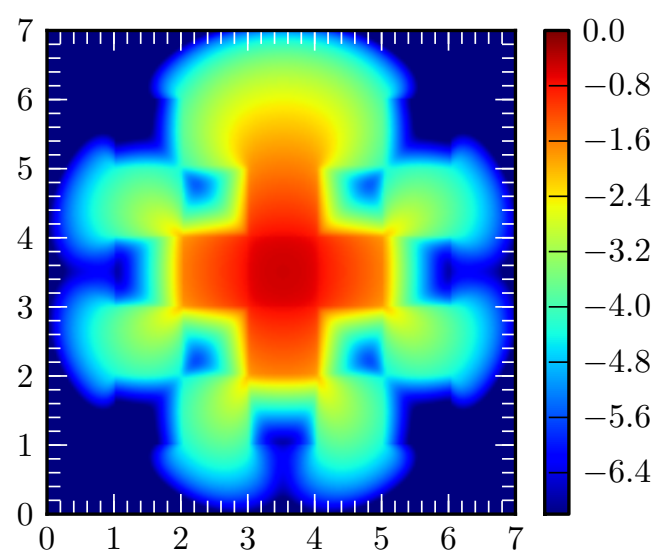

(f) $F P_{7}$ solution

Figure 8: Upper-left panel: illustration of the setup of the 2D lattice problem. The rest of the panels: $\operatorname{colormap}$ of the $\log _{10}$ of the radiation energy density at time $t=3.2 / \mathrm{c}$ as obtained with the $P_{7}$ scheme (upper-right panel) and $F P_{N}$ schemes with the Lanczos filter with opacity 5 for different order $N$ (middle and bottom panels). The time $t=3.2 / c$ corresponds to the moment when the radiation front first reaches the outer boundary of the computational domain. 
but where the radiation wavefront has reached only half of the computational domain. This is again due to the fact that the $N=1$ wave travels at a slower velocity of $\simeq c / \sqrt{3}$ [39] (see the discussion in Section 3.2). The middle-right panel shows the $F P_{3}$ solution, which also does not have regions with negative energy density and where the front has travelled enough to cover $\sim 95 \%$ of the computational domain, as a result of larger propagation speeds with higher $N$ (of course, the velocity is always bounded by the speed of light). The $F P_{5}$ solution shown in the lower-left panel is very similar to the $F P_{3}$ case, with the only noticeable difference being the slightly faster propagation velocity in the $F P_{5}$ case. Finally, the lower-right panel shows the $\mathrm{FP}_{7}$ solution, which looks almost indistinguishable from the $\mathrm{FP}_{5}$ one.

We complete the analysis of this test by showing in Fig. 9 equivalent snapshots of the energy density at a later time of $t=16 / c$, when the radiation field has reached a stationary state. In these conditions, the $P_{N}$ solution is not expected to have any negative values [9. This is indeed confirmed by the upper left panel of Fig. 9, which shows the $P_{7}$ solution without negative regions. The remaining panels in Fig. 9 report the $F P_{1}, F P_{3}$, and $F P_{7}$ solutions, respectively. Note that the $F P_{3}$, and $F P_{7}$ solutions are very similar to the $P_{7}$ one, underlining that the filter we use does not compromise the accuracy of the solution. However, the $F P_{1}$ solution appears to be significantly different from the $F P_{3}$ and $F P_{7}$ solutions, implying that $N=1$ is not a sufficiently accurate approximation for this problem.

\subsection{D Homogeneous sphere}

Finally, we consider the 3D homogeneous sphere problem, which is frequently employed to test radiation transport codes [45, 43, 3. This problem consists of a static homogeneous and isothermal sphere of radius $R$ that radiates in vacuum. Inside the sphere, the radiation interacts with the background matter only via isotropic absorption and thermal emission. Despite the rather simple setup, the sharp discontinuity at the surface of the sphere is a model for astrophysical phenomena with rapidly varying opacity. This represent a major challenge for finite-difference methods (although, it is less challenging for Monte Carlo methods; see, e.g., [3]).

We assume that the sphere of radius $R$ has a constant absorption opacity $\kappa_{a}$ and emissivity $B$ in the interior, while in the ambient vacuum at $r>R$, we have $\kappa_{a}=B=0$. For this problem, the transport equation can be solved analytically and has solution 45

$$
I(r, \mu)=\left.\frac{B}{\kappa_{a}}\right|_{r=0}\left[1-\exp \left(-\kappa_{a} s(r, \mu)\right)\right],
$$

where $r \equiv \sqrt{x^{2}+y^{2}+z^{2}}, \mu \equiv \cos \theta$ and

$$
s(r, \mu)= \begin{cases}r \mu+R g(r, \mu) & \text { if } r<R, \quad-1 \leq \mu \leq 1, \\ 2 R g(r, \mu) & \text { if } r \geq R, \quad \sqrt{1-(R / r)^{2}} \leq \mu \leq 1, \\ 0 & \text { otherwise, }\end{cases}
$$

and

$$
g(r, \mu)=\sqrt{1-\left(\frac{r}{R}\right)^{2}\left(1-\mu^{2}\right)} .
$$

Note that this solution depends only on three parameters: $\kappa_{a}, R$, and $B$, where the latter acts as a scale factor for the solution.

We perform simulations in full 3D with Cartesian coordinates and use the following computational setup. We set $R=1$ and cover the interior of the sphere with 40 elements in diameter along each coordinate direction, with the outer boundary being located at $5 R$. The absorption opacity is chosen to be $\kappa_{a}=10$ and the CFL factor is set to be $\sim 0.12$.

It is useful to remark that, although the matter distribution is spherically symmetric, this is a genuinely $3 \mathrm{D}$ test due to the Cartesian geometry of our spatial grid. Indeed, it leads to the propagation of radiation 


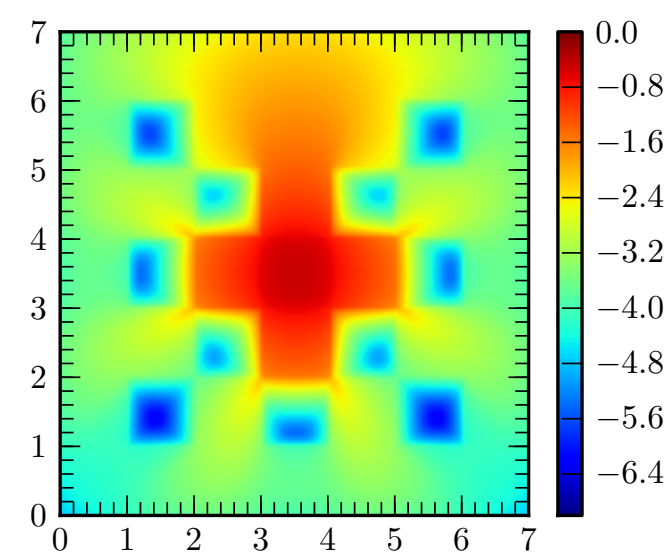

(a) $P_{7}$ solution

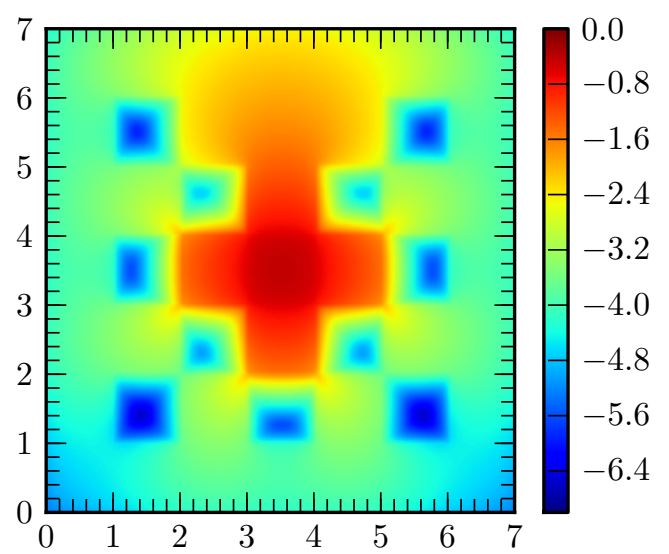

(c) $\mathrm{FP}_{3}$ solution

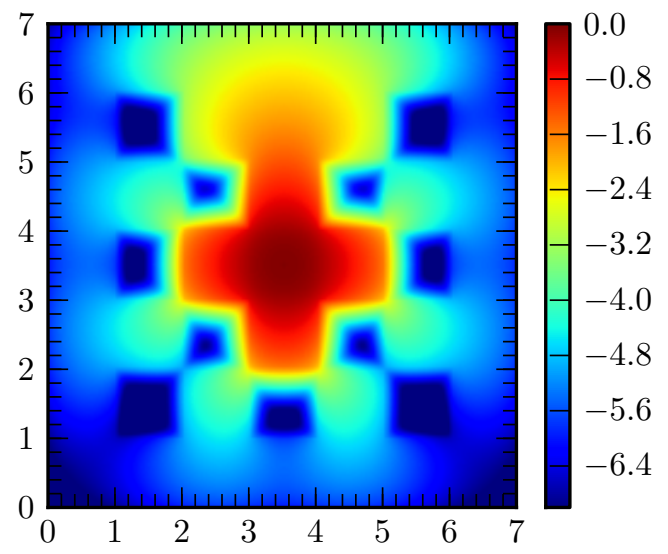

(b) $F P_{1}$ solution

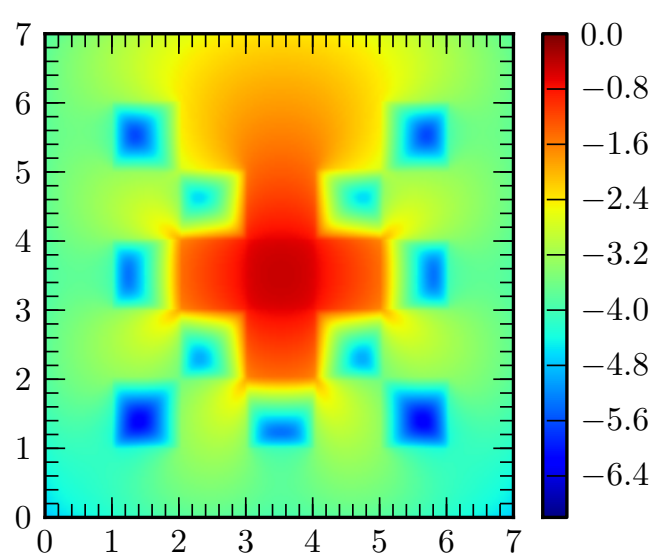

(d) $\mathrm{FP}_{7}$ solution

Figure 9: Colormaps of the $\log _{10}$ of the radiation energy density at time $t=16 / c$ obtained with the $P_{7}$ scheme (upper-left panel) and $F P_{1}$ (lower-left panel), $F P_{7}$ (upper-right panel), and $F P_{3}$ (lower-right panel) schemes with the Lanczos filter with opacity 5 . The time $t=16 / c$ corresponds to the time by which the radiation field reaches the stationary state. Since the $P_{N}$ scheme is less likely to exhibit negative solutions in the stationary state, we do not observe such solutions in our numerical result (upper-left panel), similarly to the filtered $P_{N}$ solutions. 


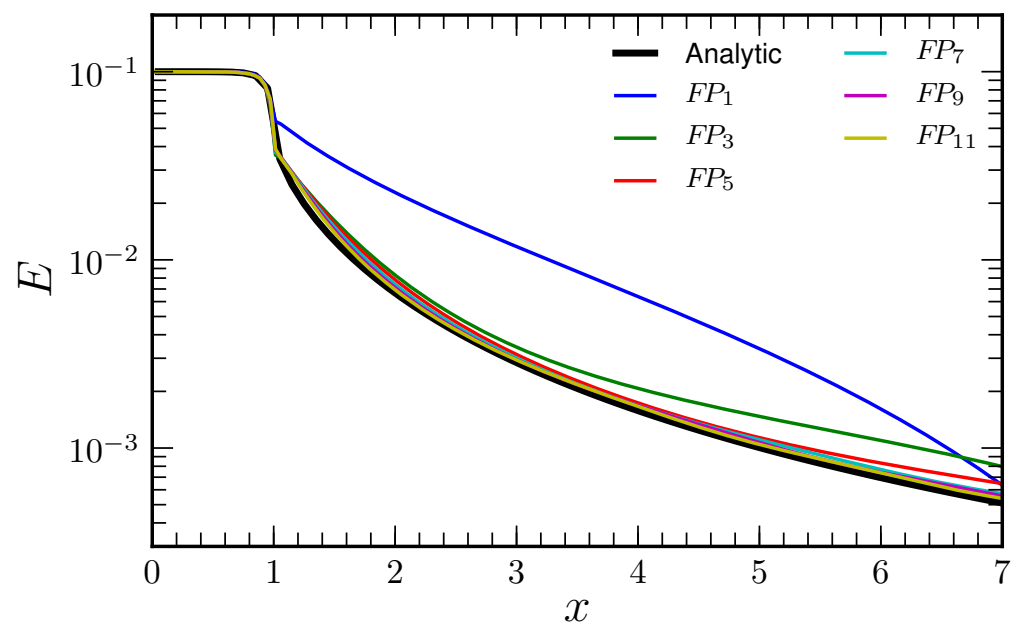

Figure 10: Radiation energy density as a function of the radial coordinate for the homogeneous sphere problem. The thick black line shows the analytical solution, while the rest of the lines show the $F P_{N}$ solution with the Lanczos filter with $\sigma_{\text {eff }}=1$ but with different values of order $N$. Clearly, the numerical result approaches the analytical solution as we increase $N$.

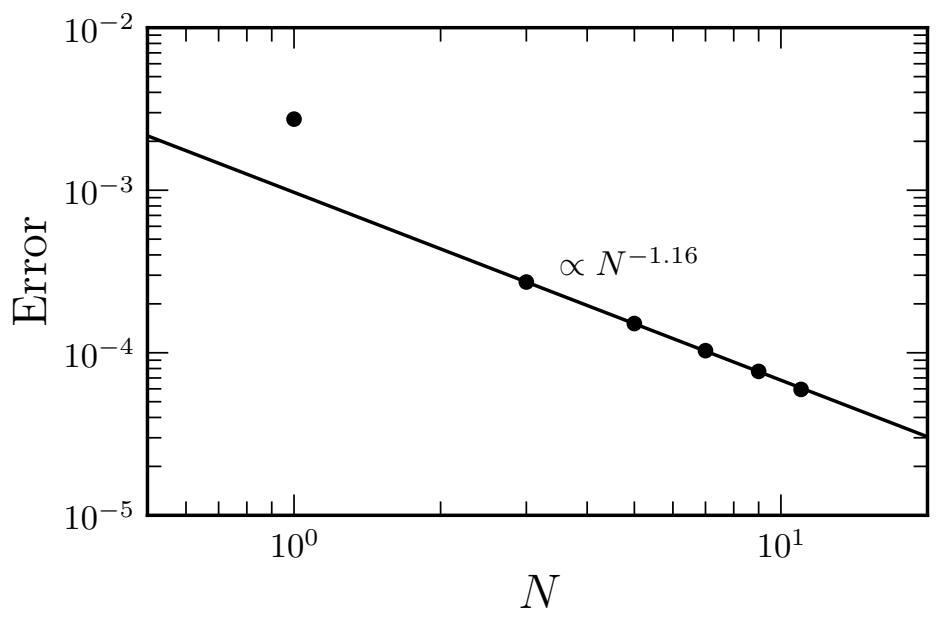

Figure 11: $L^{1}$-norm of the deviation of the $F P_{N}$ solution from the analytic result as a function of the order $N$ for the homogeneous sphere problem. The black dots show the error as computed in a sphere of radius $R=4.5$, while the black line shows the fitted convergence rate. 


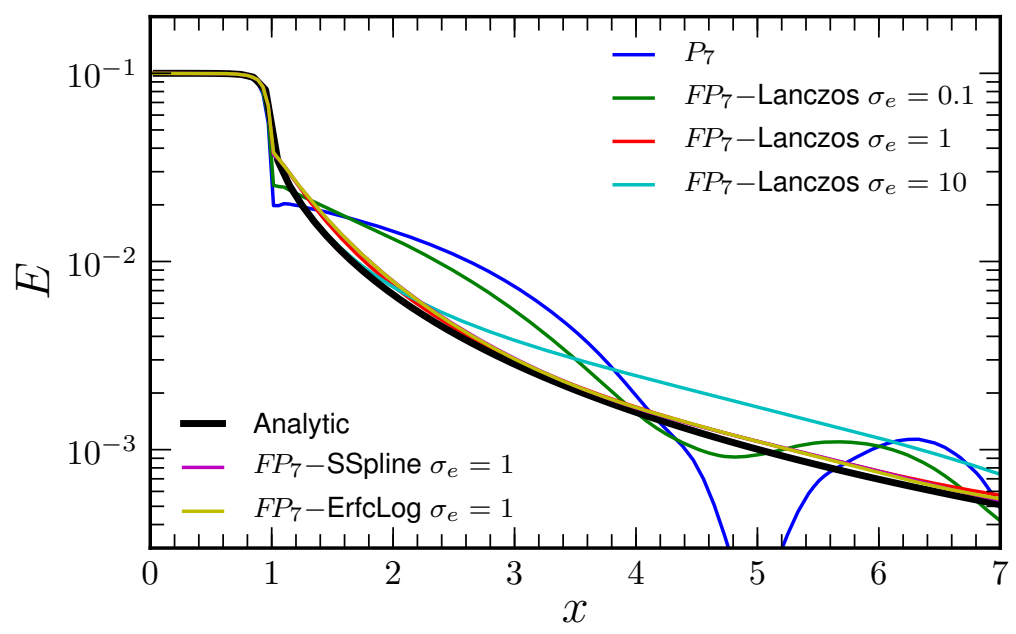

Figure 12: Radiation energy density as a function of the $r$ coordinate for the homogeneous sphere problem. The thick black line shows the analytical solution, the blue line corresponds to the unfiltered $P_{7}$ solution, while the rest of the lines represent the $\mathrm{FP}_{7}$ solutions obtained with different filters and different values of $\sigma_{\text {eff }}$.

from one spatial zone to another not only in the radial direction, but also in the angular directions, and the degree of sphericity of the numerical solution can be taken as a measure of the accuracy.

Figure 10 shows the radiation energy density along the diagonal direction for the analytical solution and the $F P_{N}$ solutions of different orders ranging from 1 to 11. The results are shown for the time when the radiation field has reached a stationary state 12 These runs are performed with the Lanczos filter of $\sigma_{\text {eff }}=1$. Interestingly, all of the $F P_{N}$ solutions produce the correct result in the interior of the sphere. This is not surprising since the radiation inside the sphere is nearly isotropic and the low-order $F P_{N}$ solutions are already accurate enough. Outside the sphere, radiation streams freely outwards with a highly forwardpeaked distribution in angle, which is a challenge for low-order $F P_{N}$ schemes. Indeed, the $F P_{1}$ result deviates significantly from the analytical solution in that region. However, the solution clearly becomes more accurate everywhere in the computational domain as we increase the order of the scheme. Figure 11 shows the $L^{1}$-norm of the deviation of the $F P_{N}$ solution from the analytic result in a sphere of radius $R=4.5{ }^{13}$ As we can see from the plot, the $F P_{N}$ scheme starts to converge already for $N \approx 3$, an order with only $4^{2}=16$ angular degrees of freedom. The convergence order is $\simeq 1.16$, which is consistent with what expected from the theory of spectral filtering.

Figure 12 shows the radiation energy density along the diagonal direction for the analytical solution and different numerical solutions at stationarity state. These are the unfiltered $P_{7}$ solution, the three $\mathrm{FP}_{7}$ solutions computed using respectively the second-order Lanczos, the fourth-order spherical-spline and ErfcLog filters with $\sigma_{\text {eff }}=1$. Also shown are the two $\mathrm{FP}_{7}$ solutions computed using the Lanczos filter with $\sigma_{\text {eff }}=0.1$ and $\sigma_{\text {eff }}=10$. Although the $P_{7}$ solution does not exhibit any negative solutions, it shows large oscillations in the free streaming region. The $\mathrm{FP}_{7}$ solution with the Lanczos filter with $\sigma_{\text {eff }}=0.1$ also yields a somewhat oscillatory solution, suggesting that the filter effective opacity is too low for this problem. As $\sigma_{\text {eff }}$ is increased, the spurious oscillations disappear and all the filters that we have tried yield solutions of very similar quality for $\sigma_{\text {eff }}=1$. Finally, the $F P_{7}$ solution with the Lanczos filter with $\sigma_{\text {eff }}=10$ is similar in quality to the $F P_{3}$ solution with the same filter but with $\sigma_{\text {eff }}=1$. This is due to the excessive damping of the high-order multiples of the solution by the filtering procedure.

\footnotetext{
${ }^{12}$ Note that stationarity is reached at different times depending on the different schemes used. For this reason and given the high computational costs, we did not evolve all the models up to the same time. Instead we report the solution as obtained as soon as stationarity is reached. In all cases the computations are performed up to at least $t=20 / c$.

${ }^{13}$ We compute the error inside $R=4.5$ instead of $R=5$ in order to exclude effects due to boundary conditions. We also normalize the $L^{1}-$ norm by dividing it by $\frac{4}{3} \pi R^{3}$.
} 


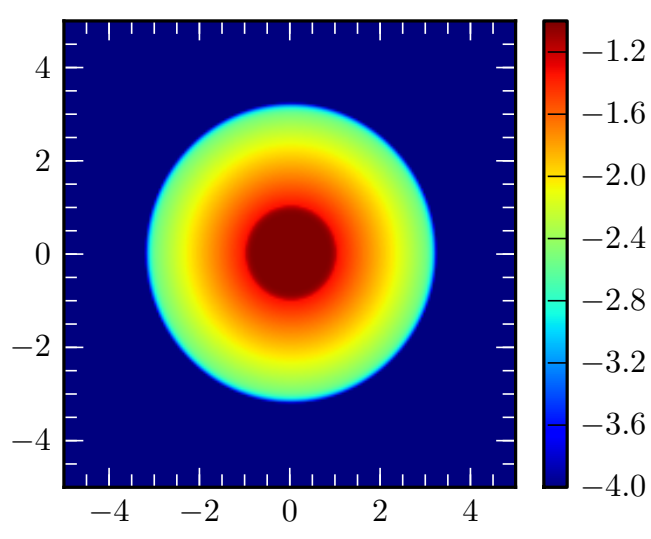

(a) $F P_{1}$ solution

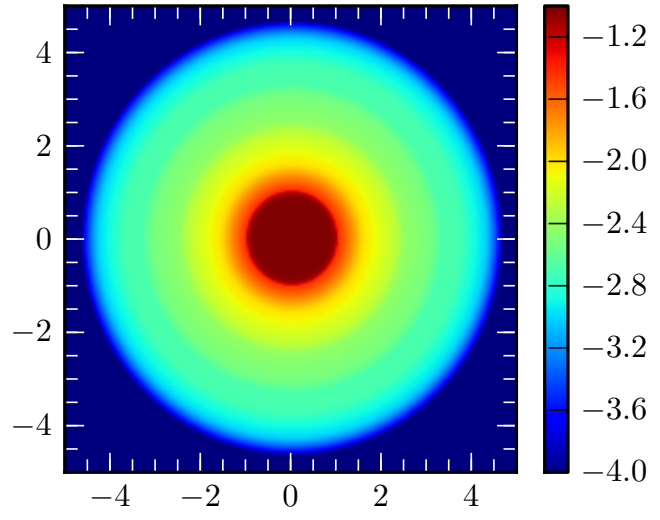

(b) $F P_{11}$ solution

Figure 13: Colormaps of the radiation energy density at time $t=3.75 / c$ obtained with the $F P_{1}$ and $F P_{7}$ schemes using the Lanczos filter with opacity $\sigma_{\text {eff }}=1$. The time $t=3.75 / c$ corresponds to the moment when the radiation front almost reaches the outer boundary of the computational domain.

Finally, Fig. 13 shows the colormaps of the $\log _{10}$ of the radiation energy density from the $F P_{1}$ (left panel) and $F P_{11}$ (right panel) solutions with the Lanczos filter with $\sigma_{\text {eff }}=1$ at $t=3.75 / c$, which corresponds to the time when the radiation front almost reaches the outer boundary. Clearly, and as observed also in the previous tests, the the $F P_{1}$ radiation front lags significantly behind the $F P_{11}$ one because of its slower propagation speed (cf. the discussion in Section 3.6). Moreover, we can also see that both solutions maintain a high level of spherical symmetry despite the Cartesian geometry of the spatial grid. We obtain similar results in tests where the sphere was covered by 20, 10 and 5 elements.

\section{Conclusion}

We have presented an extension of the filtered spherical harmonics method by McClarren and Hauck 31], the $F P_{N}$ scheme, to three dimensions. We have developed the new $3 \mathrm{D} / \mathrm{multigroup}$ radiation transport code Charon, built within the Cactus Computational Toolkit [23, 1]. Charon uses an asymptoticpreserving linear discontinuous Galerkin discretization scheme in space [33] and a semi-implicit time integration scheme 30 (cf. Section 3).

Our filtering scheme differs from the one presented by 31 in one important aspect: we reformulate the filtering procedure so that it acquires a well-defined continuum limit. In particular, we have shown that in the limit where the spatial and time steps are reduced to zero, our filtering scheme can be interpreted as the addition of a forward-peaked artificial scattering term to the $P_{N}$ equations. The filtering procedure is also constructed in such a way as to retain the convergence of the $F P_{N}$ solution to the solution of the transport equation as $N \rightarrow \infty$.

We have tested our scheme against a few challenging benchmark problems for radiation transport using four different filtering kernels: the fourth order spherical-spline filter, which is similar in spirit to the filter used by [31, the fourth-order and second-order ErfcLog filters [7, and the classical second-order Lanczos filter [7. Our findings indicate that the $F P_{N}$ scheme behaves well also in the three-dimensional case. In addition, we have shown that the second-order filters are more robust and accurate and require somewhat less tuning of the filter strength when compared to the fourth-order spherical-spline and the ErfcLog filters. Since the order of a filter is one of its most important properties, this result is likely to apply also to several other second- and fourth-order filters.

In future work, we plan to extend our numerical algorithms to include velocity dependence, the coupling to hydrodynamics, and, eventually, general relativity. 


\section{Acknowledgments}

We are happy to acknowledge helpful exchanges with Adam Burrows, Filippo Galeazzi, Cory D. Hauck, Ryan G. McClarren, Christian Reisswig and Erik Schnetter. This work was supported in part by NSF under grant nos. AST-0855535, OCI-0905046, by the Sherman Fairchild Foundation and the Alfred P. Sloan Foundation, by the DFG grant SFB/Transregio 7, and by "CompStar" a Research Networking Programme of the European Science Foundation. Results presented in this article were obtained through computations on the AEI computer cluster "Datura", on machines of the Louisiana Optical Network Initiative under grant loni_numrel07, on the Caltech compute cluster "Zwicky" (NSF MRI award No. PHY-0960291), on the NSF XSEDE network under computer time grant TG-PHY100033, and at the National Energy Research Scientific Computing Center (NERSC), which is supported by the Office of Science of the US Department of Energy under contract DE-AC03-76SF00098. This work was initiated at the MICRA workshop at the Perimeter Institute in 2011.

\section{Appendix A. Real spherical harmonics}

This Appendix is dedicated to the derivation of the real spherical harmonics, whose implementation in Charon has been particularly advantageous. We start by recalling that the spherical harmonics, $Y_{\ell}^{m}$ are the eigenfunctions of the Laplace-Beltrami operator, $\triangle$, on the unit 2-sphere:

$$
\triangle Y_{\ell}^{m}=-\ell(1+\ell) Y_{\ell}^{m} .
$$

Spherical harmonics are usually written, in complex form, as

$$
Y_{\ell}^{m}(\varphi, \theta)=e^{i m \varphi} P_{\ell}^{m}(\cos \theta),
$$

where $-\ell \leq m \leq \ell, P_{\ell}^{m}$ are the associated Legendre function, see e.g. [8],

$$
P_{\ell}^{m}(x)=\left(1-x^{2}\right)^{m / 2} C_{\ell-m}^{m+1 / 2}(x), \quad m \geq 0,
$$

and $C_{n}^{\alpha}$ are the Gegenbauer polynomials of index $\alpha$ and degree $n$. We, also, use the standard convention that

$$
P_{\ell}^{-m}(x)=(-1)^{m} \frac{(\ell-m) !}{(\ell+m) !} P_{\ell}^{m}(x) .
$$

The associated Legendre functions of index $m \geq 0$ are orthogonal in $[-1,1]$ with unit weight [4],

$$
\int_{-1}^{1} P_{\ell}^{m}(x) P_{\ell^{\prime}}^{m}(x) \mathrm{d} x=\frac{2(\ell+m) !}{(2 \ell+1)(\ell-m) !} \delta_{\ell \ell^{\prime}},
$$

while the associated Legendre functions of degree $\ell$ and index $m, m^{\prime} \geq 0$ are orthogonal in $[-1,1]$ with weight $\left(1-x^{2}\right)^{-1}[4$,

$$
\int_{-1}^{1} P_{\ell}^{m}(x) P_{\ell}^{m^{\prime}}(x) \frac{\mathrm{d} x}{1-x^{2}}=\frac{(\ell+m) !}{m(\ell-m) !} \delta_{m m^{\prime}} .
$$

The spherical harmonics with index $m, m^{\prime} \geq 0$ are then orthogonal with unit weight on the sphere:

$$
\begin{aligned}
\int_{\mathcal{S}_{1}} Y_{\ell}^{m}(\varphi, \theta) Y_{\ell^{\prime}}^{m^{\prime}}(\varphi, \theta) \mathrm{d} \Omega & =\int_{0}^{2 \pi} e^{i\left(m-m^{\prime}\right) \varphi} \mathrm{d} \varphi \int_{0}^{\pi} P_{\ell}^{m}(\cos \theta) P_{\ell^{\prime}}^{m^{\prime}}(\cos \theta) \sin \theta \mathrm{d} \theta \\
& =\int_{0}^{2 \pi} e^{i\left(m-m^{\prime}\right) \varphi} \mathrm{d} \varphi \int_{-1}^{1} P_{\ell}^{m}(x) P_{\ell^{\prime}}^{m^{\prime}}(x) \mathrm{d} x= \\
& =2 \pi \frac{2(\ell+m) !}{(2 \ell+1)(\ell-m) !} \delta_{m m^{\prime}} \delta_{\ell \ell^{\prime}} .
\end{aligned}
$$


We can then redefine the spherical harmonics as

$$
Y_{\ell}^{m}(\varphi, \theta)=\sqrt{\frac{(2 \ell+1)}{4 \pi} \frac{(\ell-m) !}{(\ell+m) !}} e^{i m \varphi} P_{\ell}^{m}(\cos \theta)=N_{\ell}^{m} e^{i m \varphi} P_{\ell}^{m}(\cos \theta) .
$$

It is easy to see, that for all $m, m^{\prime}$, thanks to the normalization and the convention A.1, we have

$$
\int_{\mathcal{S}_{1}} Y_{\ell}^{m}(\varphi, \theta) Y_{\ell^{\prime}}^{m^{\prime}}(\varphi, \theta) \mathrm{d} \Omega=\delta_{m m^{\prime}} \delta_{\ell \ell^{\prime}}
$$

We can construct a real basis from the spherical harmonics by defining

$$
Y_{\ell m}= \begin{cases}\frac{1}{\sqrt{2}}\left(Y_{\ell}^{m}+(-1)^{m} Y_{\ell}^{-m}\right), & \text { if } m>0 \\ Y_{\ell}^{0}, & \text { if } m=0 \\ \frac{1}{i \sqrt{2}}\left(Y_{\ell}^{-m}-(-1)^{m} Y_{\ell}^{m}\right) & \text { if } m<0 .\end{cases}
$$

Using again the fact that, $N_{\ell}^{-|m|} P_{\ell}^{-|m|}=(-1)^{|m|} N_{\ell}^{|m|} P_{\ell}^{|m|}$, we obtain

$$
\begin{array}{llrl}
Y_{\ell m}(\varphi, \theta) & =\sqrt{2} N_{\ell}^{m} \cos (m \varphi) P_{\ell}^{m}(\cos \theta), & & m>0, \\
Y_{\ell m}(\varphi, \theta) & =\sqrt{2} N_{\ell}^{|m|} \sin (|m| \varphi) P_{\ell}^{|m|}(\cos \theta), & & m<0,
\end{array}
$$

which is the wanted expression for the real spherical harmonics.

We can construct a Gaussian quadrature associated with the spherical harmonics basis as a direct product of a uniform quadrature in the $\varphi$ direction:

$$
w=\frac{\pi}{M_{\varphi}+1 / 2}, \quad \varphi_{m}=\frac{\pi}{M_{\varphi}+1} m, \quad-M_{\varphi} \leq m \leq M_{\varphi} .
$$

which is accurate to order $4 M_{\varphi}+2$ [8] and a Gauss-Legendre grid in the $\theta$ direction [13]:

$$
\mu=\cos \theta, \quad w_{\ell}=\frac{2}{\left(1-\mu_{\ell}^{2}\right)\left[P_{M_{\theta}+1}^{\prime}\left(\mu_{\ell}\right)\right]^{2}}, \quad \mu_{\ell}=\left\{\mathcal{Z}\left[P_{M_{\theta}+1}(\mu)\right]\right\}_{\ell}, \quad 0 \leq \ell \leq M_{\theta},
$$

where $P_{M}$ is the Legendre polynomial of degree $M$, i.e. $P_{M}=P_{M}^{0}$ and $\mathcal{Z}[p]$ denotes the set of the roots of $p$. This quadrature formula is then accurate up to order $2 M_{\theta}+1$. This means that if we want to obtain an exact representation of the scalar product of spherical harmonics up to order $N_{\theta}$ we have to choose $M_{\theta}=N_{\theta}$.

\section{References}

[1] Cactus Computational Toolkit home page, http://www.cactuscode.org/.

[2] Taflove A. and Hagness S. C. Computational Electrodynamics: The Finite-Difference Time-Domain Method, 3rd edition. Norwood, MA: Artech House, 2005.

[3] E. Abdikamalov, A. Burrows, C. D. Ott, F. Löffler, E. O'Connor, J. C. Dolence, and E. Schnetter. A New Monte Carlo Method for Time-Dependent Neutrino Radiation Transport. Astrophys. J., 755:111, August 2012.

[4] M. Abramowitz and I. A. Stegun. Handbook of mathematical functions with formulas, graphs and mathematical tables. New York: Dover, 1968.

[5] Marvin L. Adams and Edward W. Larsen. Fast iterative methods for discrete-ordinates particle transport calculations. Progress in Nuclear Energy, 40(1):3, 2002.

[6] H. A. Bethe. Supernova mechanisms. Reviews of Modern Physics, 62:801, October 1990.

[7] J P Boyd. The Erfc-Log Filter and the Asymptotics of the Euler and Vandeven Sequence Accelerations. Proceedings of the Third Internetional Conference on Spectral and High Order Methods, pages 267-276, 1996.

[8] John P. Boyd. Chebyshev and Fourier Spectral Methods (Second Edition, Revised). Dover Publications, New York, 2001.

[9] T. A. Brunner. Forms of approximate radiation transport. Sandia Report, 2002.

[10] Thomas A. Brunner and James Paul Holloway. One-dimensional riemann solvers and the maximum entropy closure. Journal of Quantitative Spectroscopy and Radiative Transfer, 69(5):543 - 566, 2001. 
[11] Thomas a. Brunner and James Paul Holloway. Two-dimensional time dependent Riemann solvers for neutron transport. Journal of Computational Physics, 210(1):386-399, November 2005.

[12] Adam Burrows, Timothy Young, Philip Pinto, Ron Eastman, and Todd A Thompson. A New Algorithm for Supernova Neutrino Transport and Some Applications. The Astrophysical Journal, 539(2):865-887, August 2000.

[13] C. Canuto, M.Y. Hussaini, A. Quarteroni, and T.A. Zang. Spectral Methods: Fundamentals in Single Domains. Springer, Berlin, 2006.

[14] Claudio Canuto, M. Yousuff Hussani, Alfio Quarteroni, and Thomas A. Zang. Spectral Methods in Fluid Dynamics. Springer-Verlag, New York and Berlin, 2nd printing edition, 1988.

15] Christian Cardall and Anthony Mezzacappa. Conservative formulations of general relativistic kinetic theory. Physical Review D, 68(2), July 2003.

[16] J. I. Castor. Radiation Hydrodynamics. Cambridge University Press, Cambridge, UK, November 2004.

[17] C. Cercignani and G. M. Kremer. The relativistic Boltzmann equation: theory and applications. Boston: Birkhäuser, 2002. Progress in mathematical physics; v. 22, 2002.

[18] Bernardo Cockburn. The Runge-Kutta local projection P1-discontinuous Galerkin finite element method for scalar conservation laws. Math. Model. Numer. Anal. (MAN), 25:337-361, 1991.

[19] Bernardo Cockburn and Chi-Wang Shu. Runge-Kutta discontinuous Galerkin methods for convection-dominated problems. Journal of Scientific Computing, 16(3):173, 2001.

[20] J. A. Fleck, Jr. and J. D. Cummings, Jr. An implicit monte carlo scheme for calculating time and frequency dependent nonlinear radiation transport. J. Comput. Phys., 8:313-342, December 1971.

[21] N. A. Gentile. Implicit monte carlo radiation transport in multi-physics simulations. In In Proc. International Conference on Mathematics, Computational Methods \& Reactor Physics (M \& C 2009), volume May 3-7, 2009, page 1170. American Nuclear Society, LaGrange Park, IL, 2009.

[22] William F. Godoy and Xu Liu. Parallel jacobian-free newton krylov solution of the discrete ordinates method with flux limiters for 3d radiative transfer. Journal of Computational Physics, 231(11):4257, 2012.

[23] T. Goodale, G. Allen, G. Lanfermann, J. Massó, T. Radke, E. Seidel, and J. Shalf. The Cactus Framework and Toolkit: Design and Applications. In Vector and Parallel Processing - VECPAR'2002, 5th International Conference, Lecture Notes in Computer Science, Berlin, 2003. Springer.

[24] D Gottlieb. On the Gibbs phenomenon and its resolution. SIAM review, 39(4):644-668, 1997.

[25] Jan S. Hesthaven and Robert M. Kirby. Filtering in Legendre spectral methods. Math. Comput., 77:1425, 2008.

[26] I. Hubeny and A. Burrows. A New Algorithm for Two-Dimensional Transport for Astrophysical Simulations. I. General Formulation and Tests for the One-Dimensional Spherical Case. Astrophys. J., 659:1458-1487, April 2007.

[27] H.-T. Janka, K. Langanke, A. Marek, G. Martínez-Pinedo, and B. Müller. Theory of core-collapse supernovae. Phys. Rep. , 442:38-74, April 2007.

[28] R B Lowrie and J E Morel. Methods for hyperbolic systems with stiff relaxation. International Journal for Numerical Methods in Fluids, 40(3-4):413-423, September 2002.

[29] R. G. McClarren, J. P. Holloway, and T. A. Brunner. Analytic $\mathrm{P}_{1}$ solutions for time-dependent, thermal radiative transfer in several geometries. J. Quantitative Spectroscopy and Radiative Transfer, 109:389-403, February 2008.

[30] Ryan G. McClarren, Thomas M. Evans, Robert B. Lowrie, and Jeffery D. Densmore. Semi-implicit time integration for PNPN thermal radiative transfer. Journal of Computational Physics, 227(16):7561-7586, August 2008.

[31] Ryan G. McClarren and Cory D. Hauck. Robust and accurate filtered spherical harmonics expansions for radiative transfer. J. Comput. Phys., 229(16):5597-5614, August 2010.

[32] Ryan G. McClarren, James Paul Holloway, and Thomas a. Brunner. On solutions to the Pn equations for thermal radiative transfer. Journal of Computational Physics, 227(5):2864-2885, February 2008.

[33] Ryan G. McClarren and Robert B. Lowrie. The effects of slope limiting on asymptotic-preserving numerical methods for hyperbolic conservation laws. Journal of Computational Physics, 227(23):9711-9726, December 2008.

[34] A. Meister, S. Ortleb, and Th. Sonar. On spectral filtering for discontinuous Galerkin methods on unstructured triangular grids. http://cms.uni-kassel.de/unicms/fileadmin/groups/w_180000/prep/prep0904.pdf, 2009.

[35] D. Mihalas and B. Mihalas. Foundations of radiation hydrodynamics. New York: Oxford University Press, 1984.

[36] J. E. Morel, T. A. Wareing, R. B. Lowrie, and D. K. Parsons. Analysis of ray-effect mitigating techniques. Nucl. Sci. Eng., 144:1, 2003.

[37] Gordon L. Olson. Second-order time evolution of pn equations for radiation transport. J. Comput. Phys., 228(8):30723083, May 2009.

[38] Gordon L. Olson. Alternate closures for radiation transport using legendre polynomials in $1 \mathrm{~d}$ and spherical harmonics in 2d. J. Comput. Phys., 231(7):2786-2793, April 2012.

[39] Gordon L. Olson, Lawrence H. Auer, and Michael L. Hall. Diffusion, p1, and other approximate forms of radiation transport. Journal of Quantitative Spectroscopy and Radiative Transfer, 64(6):619-634, 2000.

[40] C. D. Ott, A. Burrows, L. Dessart, and E. Livne. Two-Dimensional Multiangle, Multigroup Neutrino RadiationHydrodynamic Simulations of Postbounce Supernova Cores. Astrophys. J., 685:1069-1088, October 2008.

[41] G. C. Pomraning. The equations of radiation hydrodynamics. Oxford: Pergamon Press, 1973.

[42] D. Radice and L. Rezzolla. Discontinuous Galerkin methods for general-relativistic hydrodynamics: Formulation and application to spherically symmetric spacetimes. Phys. Rev. D, 84(2):024010, July 2011.

[43] M. Rampp and H.-T. Janka. Radiation hydrodynamics with neutrinos. Variable Eddington factor method for core-collapse supernova simulations. Astron. Astrophys. , 396:361, December 2002.

[44] M. Ruffert and H.-Th. Janka. Colliding neutron stars, gravitational waves, neutrino emission, and gamma-ray bursts. Astron. Astrophys., 338:535-555, 1998. 
[45] J. M. Smit, J. Cernohorsky, and C. P. Dullemond. Hyperbolicity and critical points in two-moment approximate radiative transfer. Astron. Astrophys. , 325:203, September 1997.

[46] K. Sumiyoshi and S. Yamada. Neutrino Transfer in Three Dimensions for Core-collapse Supernovae. I. Static Configurations. Astrophys. J. Supp. Ser., 199:17, March 2012.

[47] Jared Tanner. Optimal filter and mollifier for piecewise smooth spectral data. Mathematics of Computation, 75(254):767791, January 2006.

[48] Hervé Vandeven. Family of spectral filters for discontinuous problems. Journal of Scientific Computing, 6(2):159-192, June 1991. 